\title{
The Barreiro suite in the central Ribeira Belt (SE-Brazil): a late Tonian tholeiitic intraplate magmatic event in the distal passive margin of the São Francisco Paleocontinent
}

\author{
Monica Heilbron ${ }^{1,2 *}$ (D), Caroline Oliveira' (D), Marcela Lobato' (D), \\ Claudio de Morisson Valeriano ${ }^{1,2}$ (D), Ivo Dussin' (D), Elton Dantas ${ }^{3}$ (D), \\ Antonio Simonetti ${ }^{4}$ (D), Henrique Bruno ${ }^{1}$ (D), Felipe Corrales ${ }^{1}$ (D), Eduardo Socoloff ${ }^{1}$
}

\begin{abstract}
New geochemical, U-Pb, Lu-Hf and Sr-Nd data from the Barreiro Suite metabasites in comparison with enclaves within the distal Andrelândia Group and the orthogranulites of the Juiz de Fora Complex are presented. Geochemical data suggest intraplate setting, with high and low$\mathrm{TiO}_{2}, \mathrm{TDM}_{\mathrm{Nd}}$ ages between 1.80 and $1.41 \mathrm{Ga}$, negative $\mathrm{ENd}_{\mathrm{t}}$ and $\left({ }^{87} \mathrm{Sr} /{ }^{86} \mathrm{Sr}\right) \mathrm{i}$ between 0.714 and 0.747 . Results contrast with part of the Juiz de Fora Complex enclaves, with island arc tholeiites- calcalkaline basalts (IAT-CAB) geochemical signatures, $\mathrm{TDM}_{\mathrm{Nd}}$ ages between 2.58 and $2.16 \mathrm{Ga}$, positive $\mathrm{ENd}$ values and $(87 \mathrm{Sr} / 86 \mathrm{Sr})$, between 0.700 and 0.712 . U-Pb data for the Barreiro Suite yielded a crystallization age of 766 $\pm 13 \mathrm{Ma}$ and a metamorphic overprint of $619 \pm 6 \mathrm{Ma}$. The results indicate three episodes of mafic magmatism in the Occidental terrane of the Ribeira Belt. The two older episodes are related to Rhyacian arc evolution (ca. 2.2 to $2.1 \mathrm{Ga}$ ) and to the Statherian (ca. $1.7 \mathrm{Ga}$ ) tectonics, and occur only within the Juiz de Fora Complex, while the younger ca. $766 \mathrm{Ma}$ episode constrains the timing of distal passive margin evolution. An important implication is that these late Tonian metabasic rocks could have been a source of detrital zircons for the sedimentation along the distal Andrelândia basin.
\end{abstract}

KEYWORDS: Andrelândia Group; U-Pb; Sm-Nd; Lu-Hf; within-plate basalts.

\section{INTRODUCTION}

Tholeiitic mafic rocks are very common in many different tectonic environments, ranging from continental and oceanic plateaus, continental rifts to passive margins and convergent tectonic settings such as magmatic arcs (Pearce \& Cann 1973, Pearce $e$ al. 1975, Pearce 1983, 1987, Wilson 1989). In high-grade metamorphic terrains, the original tectonic setting is commonly unclear because recrystallization and deformation obscure primary characteristics. In such terrains, the geochemical and isotopic signatures, and the age of mafic rocks are important tools for the determination of their tectonic setting. Geochronological dating also provides additional constraints on the age of genetically-related high-grade metasedimentary units in orogenic belts.

The Ribeira Belt, southeast Brazil, comprises a Neoproterozoic and Early Paleozoic network of orogenic belts that resulted from the amalgamation of Western Gondwana (Fig. 1A),

${ }^{1}$ Universidade do Estado do Rio de Janeiro - Rio de Janeiro (RJ), Brazil. E-mails: monica.heilbron@gmail.com, caroline.oliveira.geo@gmail.com, marcelalobato@gmail.com, valeriano.claudio@gmail.com, ivodusin@yahoo.com.br, henrique.bruno1602@gmail.com, eduardo.socoloff@gmail.com

${ }^{2}$ University of Salzburg - Salzburg, Austria.

${ }^{3}$ Universidade de Brasilia - Brasília (DF), Brazil. E-mail: elton@unb.br

${ }^{4}$ University of Notre Dame - South Bend (IN), USA.

E-mail: antonio.simonetti.3@nd.edu

${ }^{*}$ Corresponding author. evolving through episodes of diachronous tectonic accretions and continental collisions in Neoproterozoic and Cambrian times. Together with the Araçuaí Belt (Heilbron et al. 2004, 2008, 2017a, Pedrosa-Soares et al. 2008, Alkmim et al. 2017), the Ribeira Belt makes up a $300 \mathrm{~km}$ wide and $600 \mathrm{~km}$ long deeply eroded orogenic system that runs roughly parallel to the southeastern coast of Brazil (Fig. 1B).

The external zone of this orogenic system comprises the reworked Neoproterozoic passive margin of the São Francisco paleocontinent. Because of intense deformation and metamorphism related to the Brasiliano collage, the metasedimentary units together with syn-depositional magmatic rocks crop out as high-grade gneisses, tectonically interleaved with highly-deformed basement slivers during the collisional episodes.

The Macaúbas and Andrelândia groups (Paciullo et al. 2000, Pedrosa Soares et al. 1998, 2000) represent the main Neoproterozoic passive margin sedimentary sequences of the Araçuaí and Ribeira belts. They are mainly siliciclastic, and depositional age constraints are mostly based on $\mathrm{U}-\mathrm{Pb}$ ages of detrital zircons from quartzitic rocks (Valeriano et al. 2004, Valladares et al. 2004, 2008, Belém et al. 2011, Westin \& Campos Neto 2013, Degler et al.2017, Frugis \& Campos Neto 2018). Results obtained suggest São Francisco craton source rocks, and the main constraint for the maximum possible sedimentation age of these units is the youngest detrital zircon, ranging in age from Tonian to Cryogenian ages. In several studies, the Cryogenian zircons are interpreted reflecting a shift from passive margin to active tectonic setting, with provenance from approaching magmatic 
arcs (Campos Neto et al. 2011, Belém et al. 2011, Degler et al. 2017). The major problem is that most of these studied units are metamorphosed to upper amphibolite to granulite facies, so many of these young zircons shows complex textures, with internal cores with different ages and compositions, suggestive of metamorphic reworking at high temperature that could lead to $\mathrm{Pb}$ loss. Therefore, another tool for constraining the depositional age of these high-grade siliciclastic rocks is the age of syn- or post-depositional magmatic rocks.

This study aims to contribute to the understanding of the development of the distal passive margin of the São Francisco paleocontinent by presenting new geological, geochemical and geochronological data for the Barreiro Suite (Heilbron et al.2012, 2017b). This recently described unit includes tholeiitic mafic rocks that occur within the distal segment of the Andrelândia basin. However, a further toll for constraining the depositional age of these high-grade siliciclastic rocks is the age of syn or post-depositional magmatic rocks. We also present a comparison of geochemical and $\mathrm{Sm}-\mathrm{Nd}$ and $\mathrm{Sr}$ isotope data from metabasic rocks intercalated in the Andrelândia and Macaúbas sequences with data for basic enclaves and layers that occur within the basement units such as the Juiz de Fora Complex.

\section{TECTONIC SETTING: THE ANDRELÂNDIA GROUP IN THE DISTAL SÃO FRANCISCO PASSIVE MARGIN}

The study area is located in the central segment of the Neoproterozoic-Cambrian Ribeira Belt (Almeida et al. 1981,
Heilbron et al. 2004). Following the tectonic subdivision of Heilbron et al. (2008, 2017a), the Ribeira Belt is divided into four tectono-stratigraphic terranes that sequentially docked against the southeastern portion of the São Francisco paleocontinental passive margin (Fig. 2). From west to east, these terranes are the Occidental Terrane (reworked SFC margin); the Paraíba do Sul-Embú Terrane, docked at ca. 620-595 Ma; the Oriental Terrane, docked at ca.605-565 Ma; and the Cabo Frio Terrane, docked at ca. 535-510 Ma.

The Occidental Terrane, the focus of this work, is regarded as the reworked passive margin of the São Francisco paleocontinent and comprises three major litho-tectonic units:

- reworked Paleoproterozoic basement and bimodal Mesoproterozoic magmatic rocks (Heilbron et al. 1998, 2010, Noce et al. 2007, Degler et al. 2018);

- a Neoproterozoic metasedimentary sequence described originally as the Andrelândia Group (Paciullo et al. 2000);

- syn- to late-collisional Neoproterozoic granitoid rocks (Fig. 2).

Studies carried out in the proximal zone of the Neoproterozoic passive margin (Paciullo 1997, Paciullo et al. 2000, Ribeiro et al. 1995) proposed a subdivision of the Andrelândia Group into two major sequences. The basal Carrancas Sequence (informal designation) comprises psammitic banded gneisses with quartzites, pelitic schist and amphibolite intercalations $(\mathrm{A} 1+\mathrm{A} 2)$, followed by a regressive succession of quartzites bearing characteristic green muscovite (A3), succeeded by a transgressive succession of graphite-rich grey
A

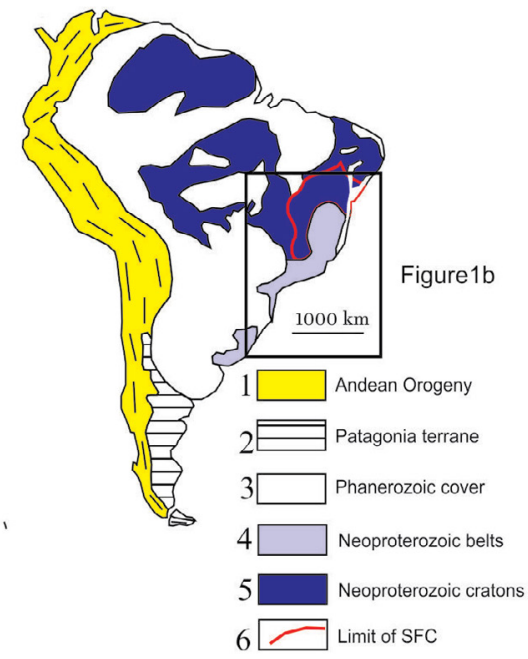

B

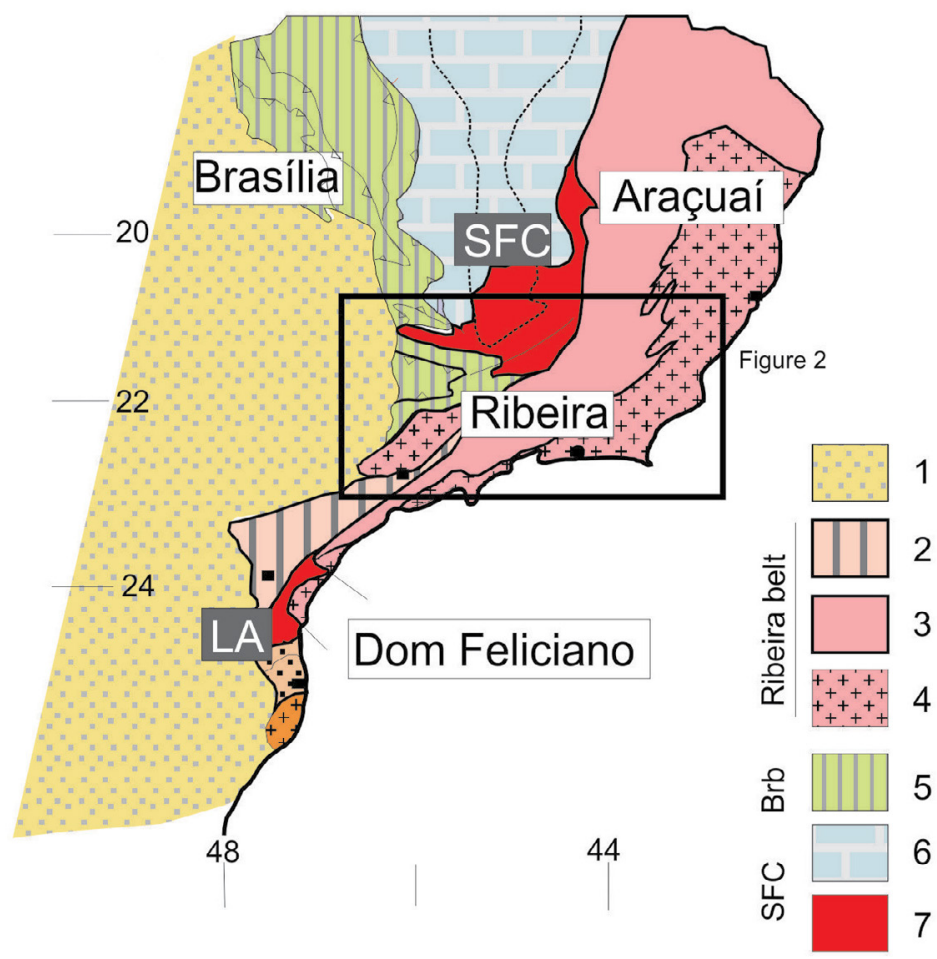

Figure 1. (A) Ribeira Belt in the scenario of South America: 1: Andean Belt; 2: Patagonia terrane; 3: Phanerozoic cover; 4: Cratons; 5: Neoproterozoic belts; 6: São Francisco craton; (B) tectonic organization of SE Brazil: 1: Phanerozoic cover; 2-4: Ribeira Belt units; 2: Apiaí terrane; 3: reworked passive margins; 4: arc-related terranes; 5: Brasília Belt. 
schists and feldspathic quartzites (A4). The overlying Serra do Turvo Sequence consists of pelitic schists and gneisses (A6), followed by the uppermost unit of plagioclase-rich schists and gneisses (A5). In the distal segment of the passive margin, where our study is located, only the basal unit (A1 + A2 equivalent?) and a pelitic unit of paragnais and schist (A6) are present. In the recent geological map of Rio de Janeiro State (Heilbron et al. 2017b), the name of Raposos Group was adopted for this distal portion of the Andrelândia Group. Because of intense tectonic shuffling with basement rocks of the Juiz de Fora Complex, and metamorphic conditions up granulite facies, the reconstruction of the architecture of this distal basin is difficult.

Major attempts to determine the depositional age of the Andrelândia Group were based on detrital zircons in metasedimentary rocks and on few Sm-Nd model ages of intercalated metabasic rocks (Heilbron et al. 1989, Frugis \& Campos Neto 2018). Most data point a Tonian onset of sedimentation after ca. 1.0-0.9 Ga (Valeriano et al. 2004, Valladares et al. 2004. On the other hand, younger Neoproterozoic zircons with ages of ca. $680 \mathrm{Ma}$ for the A5 unit have been attributed to provenance from magmatic arcs during the orogenic stage (Belém et al. 2011, Frugis \& Campos Neto 2018, Westin \& Campos Neto 2013), indicating a shift to an active setting. It is important to stress that high-pressure granulite facies metamorphism between 640 and $600 \mathrm{Ma}$ hampers precise determination of the minimum age of sedimentation (Coelho et al. 2017, Heilbron et al. 2017a, Trouw et al. 2013).
Metabasic rocks are interlayered with the $\mathrm{A} 1+\mathrm{A} 2$ and $\mathrm{A} 6$ units and normally parallel to the primary sedimentary bedding. They vary from fine-grained and foliated amphibolites to coarse-grained mafic granulites. In the high-pressure metamorphic facies, garnet + clinopyroxene + quartz is a very common assemblage in the lower Andrelândia nappe system. Relics of retro-eclogite have been described by Silva et al. (2002), Trouw et al. (2000, 2013), Campos Neto (2000), and by Coelho et al. (2017). In the upper nappe system, referred to Heilbron et al. (2000) as the Juiz de Fora crustal duplex system, (garnet) + orthopyroxene + clinopyroxene + quartz is the most common assemblage (Duarte et al. 2003).

Gonçalves \& Figueiredo (1992) studied mafic rocks that occur near the town of Andrelândia, while Marins (2000) presented geochemical data for mafic rocks located between Conservatória and Barra do Piraí. Both studies report intraplate to MORB-like signatures. A few Sm-Nd model ages of ca. 1.0 Ga were also reported for mafic rocks near Andrelândia town (Heilbron et al. 1989).

\section{MAFIC ROCKS OF THE STUDY AREA: GEOLOGY AND PETROGRAPHY}

Centimetric to decametric enclaves of metabasic rocks are interlayered within rocks of the Neoproterozoic Andrelândia Group and the orthogranulites of the Juiz de Fora Complex.

The Barreiro Suite is dominated by metabasic bodies shown in the 1:100.000 geological maps of Santo Antônio de Pádua

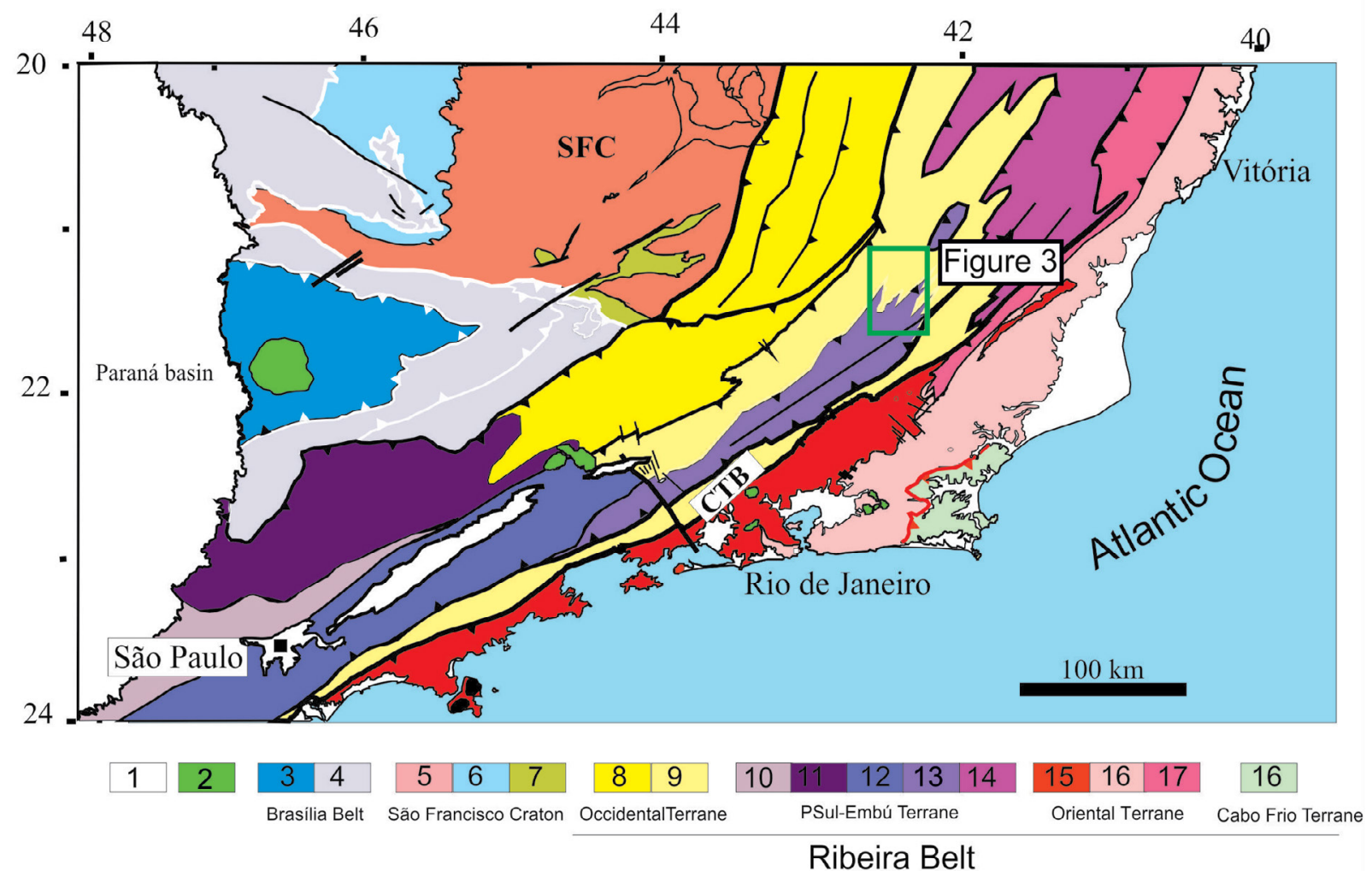

1: Phanerozoic cover; 2: K-T alkaline rocks; 3: passive margin related nappes; 4: arc-related nappes; 5: cratonic basement; 6: Bambuí cover; 7: Mesoproterozoic rift-to-sag units; 8-9: Occidental Terrane with 8 - Andrelândia (lower thrust) and 9 - Juiz de Fora (upper thrust). Cordilleran arc accreted terrane, with 10: Apiaí; 11: Socorro; 12: Embú; 13: Paraíba do Sul; 14: Rio Doce; 15: Rio Negro arc; 16: Italva arc; 17: high-grade metasediments; 18: Cabo Frio Terrane. Figure 2. Tectonic map of central Ribeira Belt, modified from Heilbron et al. (2017a). The green rectangle shows the studied area. 
(Heilbron et al.2012) and Ubá (Noce et al.2003) sheets. A decametric-scale mafic body was described within the orthogranulites of the Juiz de Fora Complex. Initially, our work was conducted to date this mafic occurrence. However, our detailed geological mapping shows that the Barreiro suite also occur within the Andrelândia metasedimentary rocks and within the ca. 620-595 Ma syn-collisional garnet charnockites (diatexites) of the Salvaterra suite (Duarte et al. 2013) (Fig. 3). The major mafic bodies occur in the western part of the mapped area, as enclaves within this syn-collision Brasiliano granitoid suite. For comparison, these minor lenses within the other country rocks were sampled.

The contact relationships with country rocks are different in the two situations. The lenses in the garnet charnockite, especially the centimentric ones, show textural evidence of partial digestion, suggesting that they are refractory material that survived partial melting.

\section{Mafic lenses within the Andrelândia Group and the Juiz de Fora Complex}

The mafic lenses (Figs. 3 and 4) have centimetre to decametre dimensions and display at least two types of relationships with country rocks. Some of the largest bodies display very sharp contacts either with the metasedimentary rocks of the Andrelândia Group, or with the orthogranulites of the Juiz de Fora Complex. On the other hand, centimetric scale enclaves within the orthogranulites show diffuse contacts possibly indicating that they have a common magmatic origin (Fig. 5).

Mafic enclaves are granulite facies rocks, containing ortho and clinopyroxene, and locally garnet (Fig. 5). Hornblende, biotite and plagioclase also occurs, besides accessory phases such as ilmenite, zircon and apatite. Textures vary from granoblastic to mylonitic, compatible with those of the country rocks.

\section{The Barreiro Suite}

In the larger (up to $50 \mathrm{~m}$ ) lenses of the Barreiro Suite (Fig. 4), two lithofacies were observed, consisting respectively of medium to fine- and coarse-grained rocks (Fig. 6). Because of the quality of the outcrops, mainly as quasi in-situ

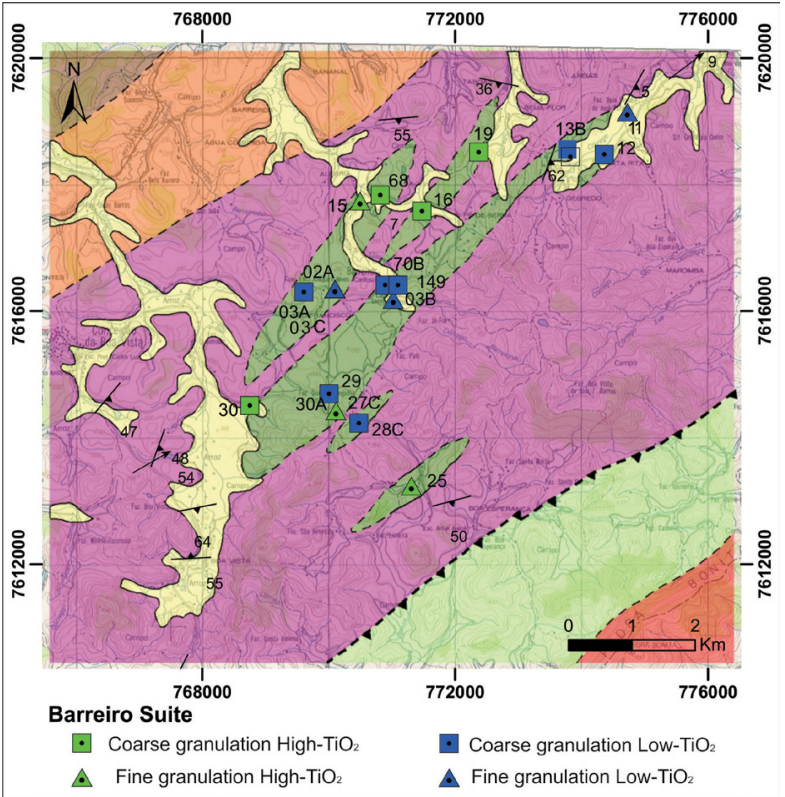

Figure 4. Geological map of the Barreiro Suite, with the location of the analysed rocks. All samples gave the same prefix code RE-CE.
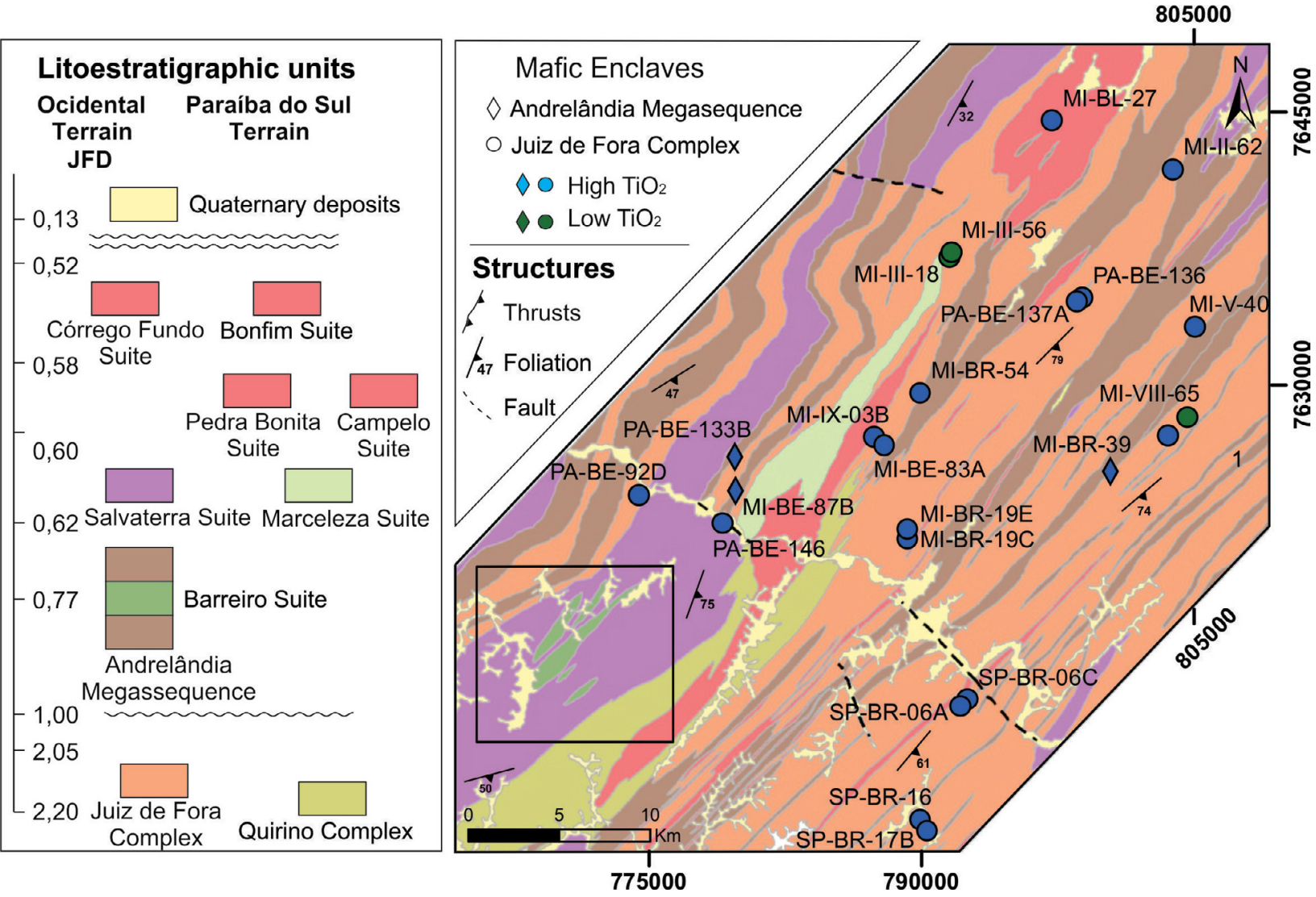

Figure 3. Geological map of the studied area with the location of the analysed enclaves from the country rocks. 
mega-boulders, it was not possible to clearly observe the relationships between these two facies. In the field, both occurs as dark gray rocks with a subtle foliation. However, under the microscope, there is a granonematoblastic texture where a visible foliation is defined by the preferential orientation of the mafic minerals. Deformational textures such as incomplete twinning of feldspars, undulose extinction and polygonal granoblastic contacts are commonly observed. The coarse-grained facies rocks locally contain plagioclase porphyroblasts, possibly representing relics of magmatic megacrysts.

The main mineralogy of both facies is represented by orthopyroxene, clinopyroxene, dark hornblende, plagioclase and opaque phases, mainly ilmenite. Biotite and hornblende also occur along the margins of the pyroxenes, suggesting formation by retrogressive reactions. Apatite, quartz and zircon are the common accessory phases.

\section{Geochemistry}

\section{Sampling and analytical procedures}

Nineteen samples of the metabasic rocks of the Barreiro suite and 23 samples of mafic lenses within Andrelândia Group and Juiz de Fora Complex rocks were selected for geochemical analyses (Tab. 1). Samples were crushed and milled at the Laboratório de Preparação de Amostras (LGPA) of the Universidade do Estado do Rio de Janeiro (UERJ). Activation Laboratories (Ontario, Canada) performed major and trace element analysis, including Rare Earth Elements (REE). Details of the analytical techniques used by this laboratory are presented at www.actlabs.com. Treatment of the data was carried out using the GeoChemical Data ToolKIT (GCDkit) software of Janoušek et al. (2006).

\section{RESULTS}

The rocks of the Barreiro suite (Tab. 1) range from gabbros to diorites, with silica contents between 47.98 and $54.48 \%$, $\mathrm{MgO}$ 2.97-7.45\%, and $\mathrm{TiO}_{2}$ 1.72-3.77. They define a subalkaline tholeiitic series that can be subdivided into high $(>2.2 \%)$ and low- $\mathrm{TiO}_{2}(<2.2 \%)$ groups (Fig. 7). Both geochemical groups are recognized in both the coarse-and fine-grained varieties of the Barreiro suite. As expected, the $\mathrm{High}-\mathrm{TiO}_{2}$ group displays relatively higher contents of $\mathrm{P}_{2} \mathrm{O}_{5}, \mathrm{REE}$ and high field strengh elements (HFSE) elements such as $\mathrm{Zr}$, Ba and $\mathrm{Y}$. The low- $\mathrm{TiO}_{2}$ group is less evolved or less contaminated as expressed by higher $\mathrm{MgO}$ and $\mathrm{CaO}$ contents and lower $\mathrm{FeO}_{\mathrm{t}}$ contents. The chondrite-normalized REE diagrams (Fig. 7) display enrichment in total content of REE and the negative $\mathrm{Eu} \backslash \mathrm{Eu}^{*}$ anomalies of the High-TiO2 group. In all tectonic discriminant diagrams (Fig. 8), both groups consistently suggest intraplate continental ( $\mathrm{CT}$ and $\mathrm{E}-\mathrm{MORB}$ ) to passive margin (E-MORB) environments, with both continental tholeiites and E-MORB signatures. The plosts involving the high-field strength elements, $\mathrm{Tb}, \mathrm{Ta}$, Th and $\mathrm{Nb}$ (Figs. $8 \mathrm{E}, 8 \mathrm{~F}$ ) seem to rule out extensional back-arc settings.

On the other hand, as expected, the mafic enclaves included in both the Andrelândia metasedimentary rocks and the orthogranulites of the Juiz de Fora Complex display
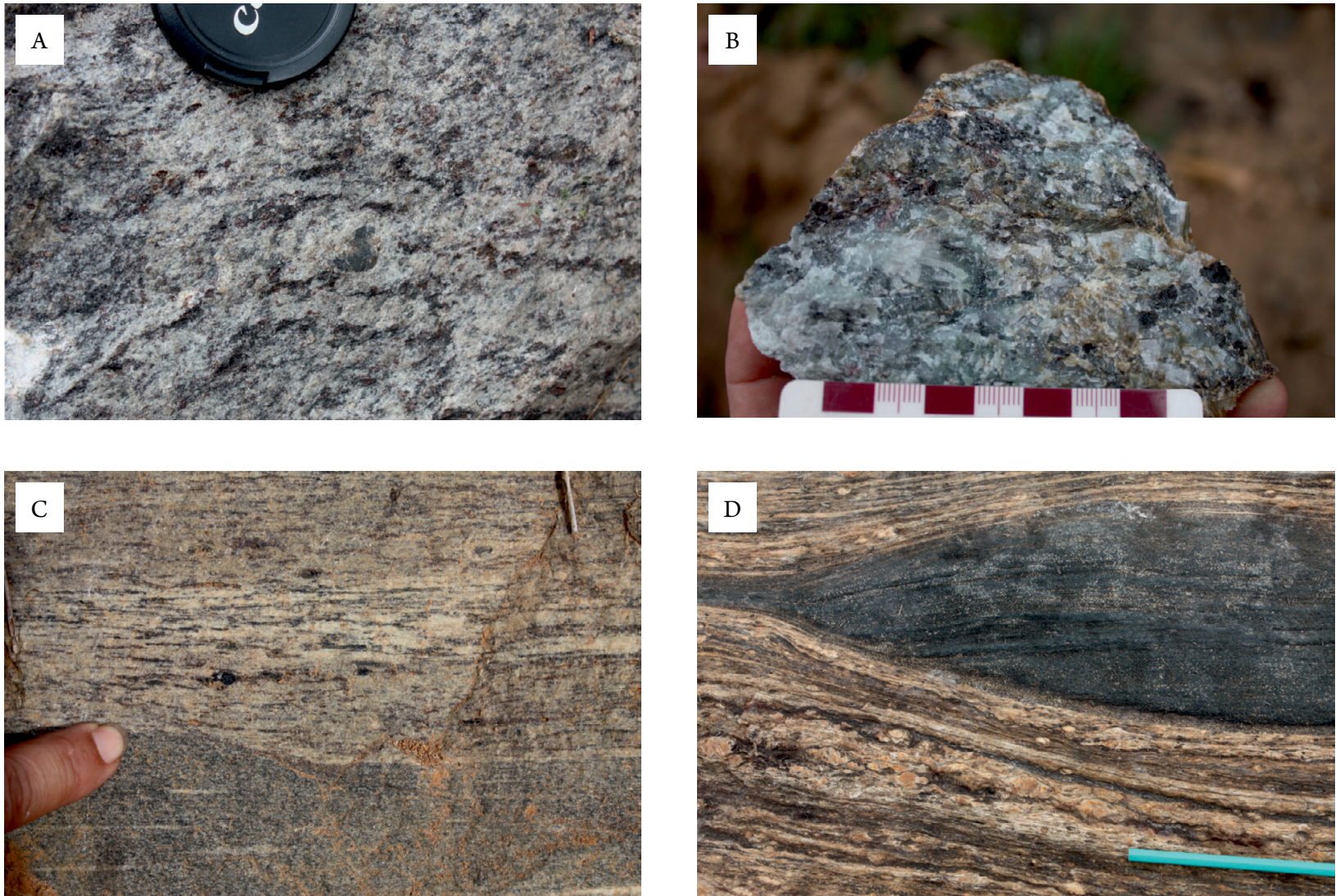

Figure 5. Outcrop images of country rocks of the Barreiro Suite. (A) Garnet-biotite gneiss of the Andrelândia Group; (B) hand sample of Opx-bearing granitoid of the Salvaterra Suite; (C) contact between the leuco-charnockite of the Juiz de Fora Complex and the grey foliated granite of the Bonfim Suite; (D) stretched mafic granulite included in the ortho-granulites of the Juiz de Fora Complex. 
heterogeneous geochemical signatures, suggesting different tectonic environments. Using the geochemical data, the enclaves of the country rocks were tentatively separated into four groups (Figs. 7 and 8):

- Group 1 with tholeiitic intraplate signatures, mostly with low- $\mathrm{TiO}_{2}$ contents, with the exception of a single sample. These enclaves occur both within the orthogranulites of the Juiz de Fora complex and in the metasedimentary rocks of the Andrelândia group. Their geochemical signature is very similar to that obtained from the low- $\mathrm{TiO}_{2}$ rocks of the Barreiro Suite;

- Group 2 comprises low- $\mathrm{TiO}_{2}$ samples with E-MORB compositions;

- Group 3 comprises intraplate alkaline mafic rocks, which occur in both the cover and basement associations;

- Group 4 represents the most common signatures for the mafic enclaves within the Juiz de Fora Complex, comprising tholeiitic to calcalkaline compositions (IAT and to
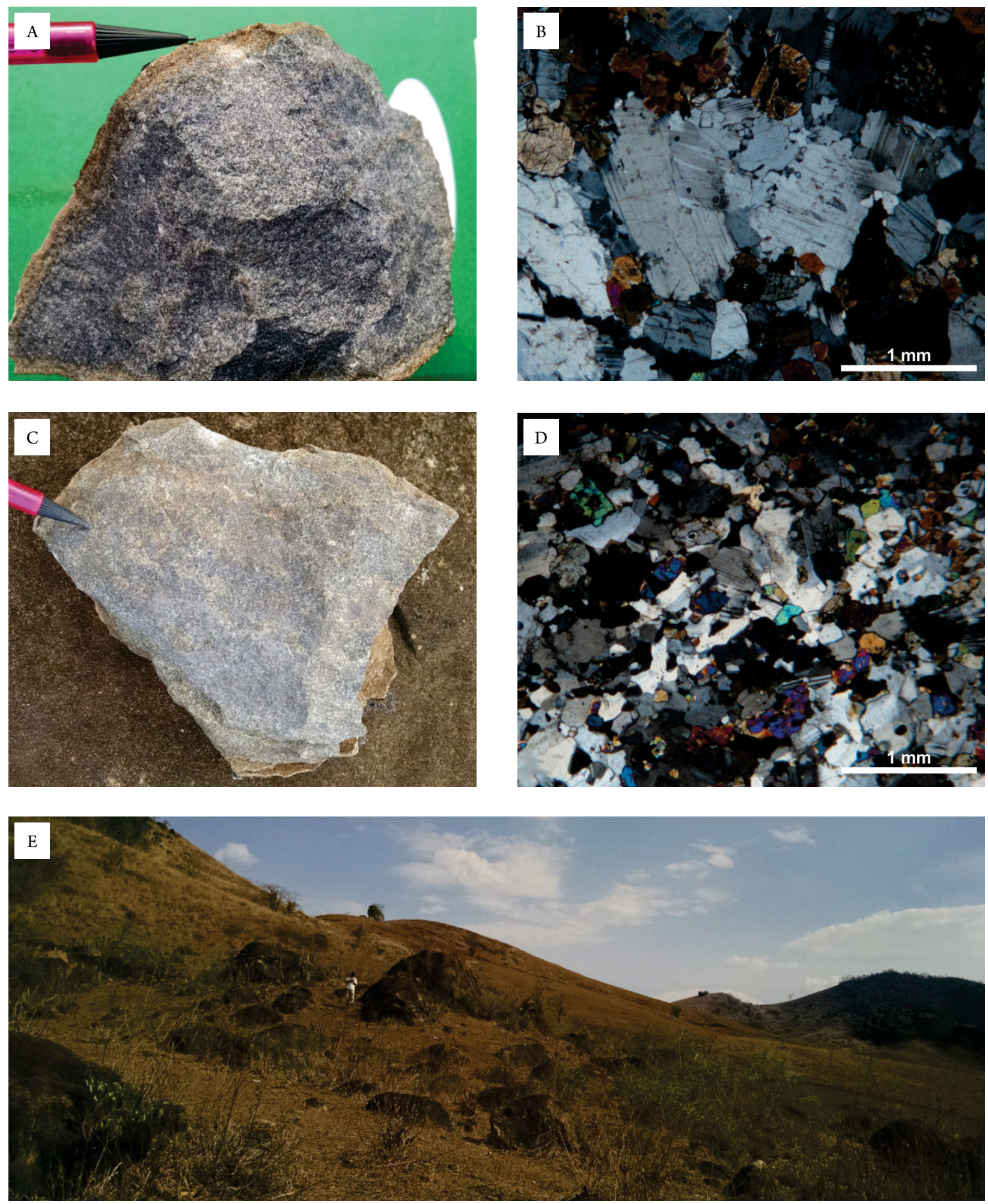

Figure 6. (A) Hand sample and (B) photomicrograph of the coarse-grained variety of metabasic rocks of the Barreiro Suite; (C) hand sample and (D) photomicrograph of the fine-grained variety of the metabasic rocks of the Barreiro Suite; (E) general overview of the Barreiro Suite outcrops with dispersed boulders. 
Table 1. Geochemical data of the metabasic rocks

\begin{tabular}{|c|c|c|c|c|c|c|c|c|c|c|c|c|c|c|c|}
\hline Sample & type & Unit & $\mathrm{SiO}_{2}$ & $\mathrm{Al}_{2} \mathrm{O}_{3}$ & $\mathrm{FeO}$ & $\mathrm{Fe2O3t}$ & MnO & MgO & $\mathrm{CaO}$ & $\mathrm{Na}_{2} \mathrm{O}$ & $\mathbf{K}_{2} \mathbf{O}$ & $\mathrm{TiO}_{2}$ & $\mathbf{P}_{2} \mathbf{O}_{5}$ & LOI & Total \\
\hline RE-CE-15 & $\mathrm{HTiO}_{2}$ & BS & 53.51 & 14.66 & 11.57 & 12.86 & 0.274 & 4.24 & 9.2 & 1.65 & 0.25 & 2.608 & 1.35 & 0.18 & 100.8 \\
\hline RE-CE-16 & $\mathrm{HTiO}_{2}$ & BS & 52.05 & 13.95 & 13.98 & 15.54 & 0.313 & 2.97 & 7.05 & 3.41 & 1.16 & 3.03 & 0.91 & -0.02 & 100.4 \\
\hline RE-CE-19 & $\mathrm{HTiO}_{2}$ & BS & 48.74 & 15.92 & 12.67 & 14.08 & 0.215 & 5.19 & 8.19 & 3.2 & 0.74 & 3.117 & 0.64 & 0.46 & 100.5 \\
\hline RE-CE-30B & $\mathrm{HTiO}_{2}$ & BS & 48.65 & 15.08 & 12.2 & 13.56 & 0.248 & 5.96 & 8.61 & 2.84 & 0.6 & 3.767 & 1.08 & -0.28 & 100.1 \\
\hline RE-CE-2A & $\mathrm{HTiO}_{2}$ & BS & 49.19 & 14.46 & 12.05 & 13.39 & 0.223 & 4.73 & 7.92 & 3.31 & 0.98 & 2.437 & 0.5 & 1.5 & 98.65 \\
\hline RE-CE-12 & $\mathrm{HTiO}_{2}$ & BS & 54.48 & 13.75 & 12.33 & 13.7 & 0.236 & 2.93 & 6.66 & 3.23 & 1.7 & 2.815 & 0.89 & -0.1 & 100.3 \\
\hline RE-CE-13B & $\mathrm{HTiO}_{2}$ & BS & 51.45 & 15.6 & 12.12 & 13.47 & 0.267 & 4.51 & 7.61 & 3.48 & 0.93 & 2.762 & 0.63 & 0.04 & 100.7 \\
\hline RE-CE-27C & $\mathrm{HTiO}_{2}$ & BS & 51.02 & 15.41 & 12.57 & 13.97 & 0.223 & 3.34 & 6.75 & 3.46 & 2.18 & 2.832 & 0.76 & 0.29 & 100.2 \\
\hline RE-CE-28C & $\mathrm{HTiO}_{2}$ & BS & 49.5 & 17.16 & 10.53 & 11.7 & 0.193 & 5.91 & 8.45 & 3.53 & 1.11 & 2.238 & 0.38 & 0.54 & 100.7 \\
\hline RE-CE-29 & $\mathrm{HTiO}_{2}$ & BS & 50.58 & 16.31 & 11.23 & 12.48 & 0.204 & 4.82 & 7.77 & 3.54 & 1.46 & 2.42 & 0.58 & 0.46 & 100.6 \\
\hline RE-CE-30A & $\mathrm{HTiO}_{2}$ & BS & 50.05 & 15.32 & 12.99 & 14.44 & 0.225 & 4.01 & 7.53 & 3.09 & 1.6 & 3.013 & 0.78 & 0.3 & 100.4 \\
\hline RE-CE-70B & $\mathrm{HTiO}_{2}$ & BS & 47.98 & 16.31 & 13 & 14.45 & .22 & 4.84 & 7.98 & 3.13 & 0.71 & 2.948 & 0.7 & -0.21 & 99.05 \\
\hline RE-CE-3A & $\mathrm{LTiO}_{2}$ & BS & 51.81 & 16.42 & 9.76 & 10.85 & 0.181 & 5.76 & 8.56 & 3.4 & 0.74 & 1.919 & 0.36 & 0.96 & 101 \\
\hline RE-CE-03B & $\mathrm{LTiO}_{2}$ & BS & 48.67 & 17.22 & 9.65 & 10.72 & 0.188 & 7.45 & 9.79 & 3.03 & 0.48 & 1.586 & 0.28 & 1.06 & 100.5 \\
\hline RE-CE-25 & $\mathrm{LTiO}_{2}$ & BS & 51.99 & 16.83 & 9.71 & 10.79 & 0.174 & 5.49 & 7.53 & 3.59 & 1.55 & 1.86 & 0.33 & 0.62 & 100.8 \\
\hline RE-CE-68 & $\mathrm{LTiO}_{2}$ & BS & 57.34 & 15.8 & 9.3 & 10.34 & 0.157 & 3.84 & 6.13 & 3.39 & 0.63 & 1.776 & 0.41 & 0.3 & 100.1 \\
\hline RE-CE-3C & $\mathrm{LTiO}_{2}$ & BS & 49.41 & 17.39 & 9.51 & 10.57 & 0.174 & 6.63 & 9.3 & 3.14 & 0.86 & 1.679 & 0.32 & 0.98 & 100.4 \\
\hline RE-CE-11 & $\mathrm{LTiO}_{2}$ & BS & 51.24 & 17.26 & 9.89 & 10.99 & 0.18 & 5.62 & 8.69 & 3.25 & 0.63 & 1.764 & 0.39 & 0.69 & 100.7 \\
\hline RE-CE-149 & $\mathrm{LTiO}_{2}$ & BS & 52.7 & 16.94 & 10.19 & 11.32 & 0.186 & 6.03 & 7.76 & 3.38 & 0.68 & 1.724 & 0.3 & -0.07 & 100.9 \\
\hline MI-BR-39 & $\mathrm{LTiO}_{2}$ & AND & 48 & 13.88 & 12.76 & 14.18 & 0.21 & 7.3 & 11.88 & 1.41 & 0.54 & 1.386 & 0.1 & -0.13 & 98.75 \\
\hline MI-BE-87B & $\mathrm{LTiO}_{2}$ & AND & 50.84 & 15.02 & 8.9 & 9.89 & 0.16 & 9.57 & 7.8 & 2.65 & 1.87 & 1.426 & 0.46 & 0.67 & 100.4 \\
\hline PA-BE-133B & $\mathrm{LTiO}_{2}$ & AND & 49.01 & 19.04 & 8.98 & 9.98 & 0.156 & 5.67 & 8.8 & 3.47 & 1.22 & 1.697 & 0.29 & 0.56 & 99.88 \\
\hline MI-BR-54 & $\mathrm{LTiO}_{2}$ & $\mathrm{JF}$ & 40.45 & 12.52 & 14.06 & 15.62 & 0.301 & 21.58 & 6.92 & 0.51 & 0.31 & 0.348 & 0.09 & 0.68 & 99.32 \\
\hline MI-VIII-15 & $\mathrm{LTiO}_{2}$ & $\mathrm{JF}$ & 46.8 & 9.98 & 9.57 & 10.64 & 0.155 & 21.07 & 8.98 & 1.28 & 0.19 & 0.335 & 0.03 & 1.28 & 100.7 \\
\hline SP-BR-16 & $\mathrm{LTiO}_{2}$ & $\mathrm{JF}$ & 48.84 & 13.79 & 13.35 & 14.84 & 0.206 & 6.64 & 10.85 & 2.78 & 0.93 & 1.435 & 0.1 & 0.28 & 100.7 \\
\hline MI-II-62 & $\mathrm{LTiO}_{2}$ & $\mathrm{JF}$ & 49.81 & 15.24 & 12.09 & 13.44 & 0.172 & 6.63 & 9.98 & 3.22 & 0.95 & 0.974 & 0.14 & 0.14 & 100.7 \\
\hline MI-V-40 & $\mathrm{LTiO}_{2}$ & JF & 46.14 & 14.95 & 11.12 & 12.36 & 25 & 8.31 & 12.6 & 2.64 & 1.17 & 1.058 & 0.28 & 1.04 & 100.8 \\
\hline MI-BL-27 & $\mathrm{LTiO}_{2}$ & $\mathrm{JF}$ & 51.01 & 14.8 & 9.37 & 10.41 & 0.167 & 7.58 & 11.9 & 1.92 & 0.65 & 0.934 & 0.12 & 0.88 & 100.4 \\
\hline PA-BE-146 & $\mathrm{LTiO}_{2}$ & JF & 50.82 & 13.84 & 10.88 & 12.09 & 0.233 & 7.76 & 10.11 & 2.69 & 0.71 & 1.146 & 0.41 & 0.11 & 99.93 \\
\hline PA-BE-136 & $\mathrm{LTiO}_{2}$ & JF & 50.85 & 15.32 & 9.75 & 10.83 & 0.163 & 7.41 & 7.6 & 3.17 & 2.39 & 1.024 & 0.48 & 0.09 & 99.31 \\
\hline SP-BR-06A & $\mathrm{LTiO}_{2}$ & $\mathrm{JF}$ & 50.07 & 14.36 & 11.58 & 12.87 & 0.185 & 7.25 & 8.52 & 2.82 & 1.19 & 0.948 & 0.23 & 1.19 & 99.65 \\
\hline PA-BE-137A & $\mathrm{LTiO}_{2}$ & $\mathrm{JF}$ & 51.27 & 10.61 & 10.13 & 11.26 & 0.203 & 12.84 & 8.85 & 2.04 & 2.32 & 0.629 & 0.39 & 0.24 & 100.7 \\
\hline SP-BR-06C & $\mathrm{LTiO}_{2}$ & $\mathrm{JF}$ & 42.48 & 18.52 & 10.95 & 12.17 & 0.138 & 6.24 & 12.23 & 2.44 & 1.44 & 1.585 & 1.05 & 0.99 & 99.28 \\
\hline SP-BR-17B & $\mathrm{LTiO}_{2}$ & $\mathrm{JF}$ & 49.22 & 14.16 & 12.62 & 14.02 & 0.187 & 7.02 & 11.23 & 2.33 & 0.68 & 1.356 & 0.11 & 0.02 & 100.3 \\
\hline MI-BR-19C & $\mathrm{LTiO}_{2}$ & JF & 48.56 & 13.65 & 13.49 & 14.99 & 0.217 & 6.59 & 10.03 & 2.64 & 1.3 & 1.557 & 0.12 & 0.69 & 100.3 \\
\hline MI-BR-19E & $\mathrm{LTiO}_{2}$ & $\mathrm{JF}$ & 51.26 & 14.41 & 12.26 & 13.62 & 0.265 & 5 & 5.28 & 2.33 & 3.35 & 1.538 & 0.13 & 1.35 & 98.93 \\
\hline PA-BE-92D & $\mathrm{LTiO}_{2}$ & $\mathrm{JF}$ & 44.68 & 12.52 & 14.75 & 16.39 & 0.247 & 11.73 & 8.2 & 1.56 & 3.7 & 0.343 & 0.04 & 1.46 & 100.9 \\
\hline MI-BE-83A & $\mathrm{LTiO}_{2}$ & $\mathrm{JF}$ & 52.16 & 1 & 9 & 1 & 0.165 & 4.99 & 7.32 & 3.64 & 1.42 & 1.621 & 0.32 & 1.2 & 99.15 \\
\hline MI-IX-03B & $\mathrm{HTiO}_{2}$ & $\mathrm{JF}$ & 51.62 & 6.41 & 9.79 & 10.88 & 0.156 & 5.22 & 7.89 & 3.15 & 1.79 & 2.157 & 0.86 & 0.27 & 100.4 \\
\hline MI-VIII-65 & $\mathrm{HTiO}_{2}$ & JF & 46.78 & 18.07 & 9.6 & 10.67 & 0.166 & 4.72 & 9 & 3.39 & 2.57 & 2.245 & 1.15 & 0.57 & 99.35 \\
\hline MI-III-18 & $\mathrm{HTiO}_{2}$ & JF & 49.11 & 16.98 & 11.45 & 12.72 & 0.2 & 5 & 8.63 & 3.23 & 0.68 & 2.467 & 0.81 & 0.07 & 99.89 \\
\hline MI-BR-56 & $\mathrm{HTiO}_{2}$ & JF & 51.05 & 16.62 & 11.4 & 12.67 & 0.201 & 4.03 & 8.09 & 3.37 & 1.48 & 2.465 & 1.13 & -0.24 & 100.9 \\
\hline
\end{tabular}


Table 1. Continuation

\begin{tabular}{|c|c|c|c|c|c|c|c|c|c|c|c|c|c|c|c|c|c|c|c|}
\hline Sample & Type & Unit & Sc & $\mathrm{Be}$ & $\mathbf{v}$ & $\mathbf{B a}$ & Sr & $\mathbf{Y}$ & $\mathbf{Z r}$ & $\mathrm{Cr}$ & Co & $\mathrm{Ni}$ & $\mathbf{C u}$ & $\mathrm{Zn}$ & Ga & Ge & As & $\mathbf{R b}$ & $\mathbf{N b}$ \\
\hline RE-CE-15 & $\mathrm{HTiO}_{2}$ & BS & 26 & 5 & 252 & 230 & 593 & 44 & 354 & 200 & 30 & 60 & 20 & 160 & 24 & 2 & 6 & 5 & 29 \\
\hline RE-CE-16 & $\mathrm{HTiO}_{2}$ & BS & 30 & 2 & 250 & 1089 & 553 & 49 & 342 & 20 & 34 & 30 & 40 & 180 & 22 & 1 & $<5$ & 8 & 8 \\
\hline RE-CE-19 & $\mathrm{HTiO}_{2}$ & BS & 30 & 1 & 262 & 657 & 610 & 34 & 174 & 30 & 44 & 30 & 40 & 120 & 21 & 1 & $<5$ & 3 & 19 \\
\hline RE-CE-30B & $\mathrm{HTiO}_{2}$ & BS & 31 & 1 & 347 & 446 & 569 & 34 & 159 & 70 & 34 & 30 & 30 & 130 & 18 & 1 & $<5$ & 7 & 23 \\
\hline RE-CE-2A & $\mathrm{HTiO}_{2}$ & BS & 27 & 2 & 225 & 775 & 507 & 39 & 287 & 20 & 39 & 20 & 30 & 140 & 21 & 2 & $<5$ & 8 & 20 \\
\hline RE-CE-12 & $\mathrm{HTiO}_{2}$ & BS & 28 & 2 & 227 & 1364 & 528 & 52 & 430 & $<20$ & 30 & $<20$ & 30 & 170 & 23 & 2 & $<5$ & 16 & 28 \\
\hline RE-CE-13B & $\mathrm{HTiO}_{2}$ & BS & 30 & 2 & 211 & 677 & 573 & 40 & 317 & 50 & 38 & 30 & 30 & 130 & 21 & 2 & $<5$ & 15 & 20 \\
\hline RE-CE-27C & $\mathrm{HTiO}_{2}$ & BS & 28 & 2 & 224 & 1605 & 498 & 50 & 545 & $<20$ & 31 & $<20$ & 30 & 140 & 23 & 1 & $<5$ & 24 & 31 \\
\hline RE-CE-28C & $\mathrm{HTiO}_{2}$ & BS & 27 & 2 & 224 & 299 & 556 & 30 & 221 & 40 & 42 & $<20$ & 30 & 90 & 20 & 1 & $<5$ & 15 & 15 \\
\hline RE-CE-29 & $\mathrm{HTiO}_{2}$ & BS & 27 & 2 & 229 & 1147 & 622 & 36 & 573 & $<20$ & 38 & 20 & 30 & 110 & 20 & 1 & $<5$ & 29 & 19 \\
\hline RE-CE-30A & $\mathrm{HTiO}_{2}$ & BS & 30 & 2 & 261 & 1377 & 553 & 48 & 510 & $<20$ & 36 & $<20$ & 30 & 140 & 22 & 1 & $<5$ & 13 & 27 \\
\hline RE-CE-70B & $\mathrm{HTiO}_{2}$ & BS & 27 & 2 & 239 & 732 & 619 & 37 & 299 & $<20$ & 45 & $<20$ & 30 & 140 & 26 & 2 & $<5$ & 6 & 20 \\
\hline RE-CE-3A & $\mathrm{LTiO}_{2}$ & BS & 26 & 2 & 207 & 466 & 626 & 20 & 153 & 70 & 37 & 30 & 40 & 100 & 20 & 1 & $<5$ & 9 & 12 \\
\hline RE-CE-03B & $\mathrm{LTiO}_{2}$ & BS & 28 & 1 & 180 & 353 & 585 & 23 & 92 & 100 & 42 & 40 & 20 & 80 & 17 & 1 & $<5$ & 3 & 9 \\
\hline RE-CE-25 & $\mathrm{LTiO}_{2}$ & BS & 25 & 2 & 187 & 437 & 537 & 30 & 225 & 40 & 37 & 30 & 30 & 90 & 19 & 1 & $<5$ & 67 & 13 \\
\hline RE-CE-68 & $\mathrm{LTiO}_{2}$ & BS & 23 & 2 & 170 & 372 & 427 & 28 & 152 & 30 & 29 & $<20$ & 20 & 100 & 25 & 2 & $<5$ & 5 & 18 \\
\hline RE-CE-3C & $\mathrm{LTiO}_{2}$ & BS & 28 & 1 & 212 & 504 & 627 & 29 & 177 & 140 & 37 & 60 & 30 & 80 & 18 & 1 & $<5$ & 9 & 11 \\
\hline RE-CE-11 & $\mathrm{LTiO}_{2}$ & BS & 27 & 2 & 220 & 711 & 646 & 27 & 257 & 30 & 37 & 30 & 30 & 100 & 19 & 1 & $<5$ & 5 & 12 \\
\hline RE-CE-149 & $\mathrm{LTiO}_{2}$ & BS & 24 & 1 & 171 & 427 & 641 & 20 & 81 & 60 & 38 & 40 & 30 & 90 & 21 & 2 & $<5$ & 6 & 3 \\
\hline MI-BR-39 & $\mathrm{LTiO}_{2}$ & AND & 41 & $<1$ & 369 & 12 & 146 & 19 & 57 & 150 & 64 & 90 & 30 & 120 & 19 & 2 & $<5$ & 7 & 4 \\
\hline MI-BE-87B & $\mathrm{LTiO}_{2}$ & AND & 26 & 2 & 186 & 1475 & 711 & 26 & 228 & 590 & 44 & 230 & 60 & 90 & 17 & 2 & $<5$ & 57 & 14 \\
\hline PA-BE-133B & $\mathrm{LTiO}_{2}$ & AND & 25 & 2 & 189 & 441 & 587 & 24 & 180 & 40 & 35 & 30 & 20 & 60 & 20 & 2 & $<5$ & 34 & 3 \\
\hline MI-BR-54 & $\mathrm{LTiO}_{2}$ & JF & 14 & $<1$ & 75 & 327 & 114 & 12 & 13 & 200 & 114 & 450 & 60 & 280 & 9 & 1 & $<5$ & 4 & 1 \\
\hline MI-VIII-15 & $\mathrm{LTiO}_{2}$ & JF & 33 & $<1$ & 186 & 22 & 35 & 19 & 20 & 2020 & 70 & 870 & $<10$ & 50 & 8 & 2 & $<5$ & $<2$ & 1 \\
\hline SP-BR-16 & $\mathrm{LTiO}_{2}$ & $\mathrm{JF}$ & 39 & $<1$ & 392 & 64 & 131 & 31 & 73 & 90 & 56 & 90 & 10 & 120 & 20 & 2 & $<5$ & 8 & 4 \\
\hline MI-II-62 & $\mathrm{LTiO}_{2}$ & JF & 36 & $<1$ & 253 & 207 & 264 & 20 & 87 & 90 & 49 & 140 & 30 & 110 & 20 & 2 & $<5$ & 5 & 4 \\
\hline MI-V-40 & $\mathrm{LTiO}_{2}$ & JF & 45 & 2 & 331 & 399 & 286 & 16 & 94 & 410 & 45 & 90 & $<10$ & 120 & 19 & 2 & $<5$ & 21 & 3 \\
\hline MI-BL-27 & $\mathrm{LTiO}_{2}$ & JF & 40 & $<1$ & 285 & 181 & 202 & $1 \varepsilon$ & 80 & 310 & 42 & 90 & 90 & 70 & 17 & 2 & $<5$ & 19 & 6 \\
\hline PA-BE-146 & $\mathrm{LTiO}_{2}$ & JF & 43 & 1 & 170 & 1164 & 642 & 32 & 102 & 400 & 35 & 110 & 40 & 100 & 18 & 2 & 5 & 16 & $<1$ \\
\hline PA-BE-136 & $\mathrm{LTiO}_{2}$ & JF & 26 & 2 & 192 & 1141 & 39 & 22 & 139 & 280 & 35 & 80 & $<10$ & 100 & 19 & 2 & $<5$ & 55 & $<1$ \\
\hline SP-BR-06A & $\mathrm{LTiO}_{2}$ & JF & 47 & 3 & 213 & 165 & 324 & 58 & 188 & 210 & 42 & 100 & 10 & 310 & 23 & 2 & $<5$ & 15 & 13 \\
\hline PA-BE-137A & $\mathrm{LTiO}_{2}$ & JF & 30 & 1 & 160 & 880 & 464 & 25 & 146 & 1000 & 42 & 260 & $<10$ & 110 & 15 & 2 & $<5$ & 72 & $<1$ \\
\hline SP-BR-06C & $\mathrm{LTiO}_{2}$ & JF & 32 & $<1$ & 389 & 256 & 765 & 34 & 60 & 30 & 50 & $<20$ & 60 & 80 & 22 & 1 & $<5$ & 32 & 5 \\
\hline SP-BR-17B & $\mathrm{LTiO}_{2}$ & JF & 40 & $<1$ & 358 & 221 & 276 & 21 & 77 & 130 & 67 & 130 & 140 & 130 & 19 & 2 & $<5$ & 4 & 4 \\
\hline MI-BR-19C & $\mathrm{LTiO}_{2}$ & JF & 43 & 1 & 419 & 134 & 250 & 30 & 8 & 110 & 54 & 80 & 30 & 150 & 22 & 2 & $<5$ & 12 & 10 \\
\hline MI-BR-19E & $\mathrm{LTiO}_{2}$ & JF & 41 & 3 & 200 & 690 & 272 & 83 & 100 & 90 & 40 & 50 & 30 & 190 & 27 & 3 & $<5$ & 235 & 47 \\
\hline PA-BE-92D & $\mathrm{LTiO}_{2}$ & JF & 27 & 3 & 1 & 352 & 30 & 2 & 32 & 7 & 54 & 420 & $<10$ & 280 & 19 & 2 & $<5$ & 157 & 9 \\
\hline MI-BE-83A & $\mathrm{LTiO}_{2}$ & $\mathrm{JF}$ & 24 & 2 & 1 & 488 & 494 & 26 & 239 & $<20$ & 33 & 2 & 20 & 90 & 19 & 2 & $<5$ & 34 & 11 \\
\hline MI-IX-03B & $\mathrm{HTiO}_{2}$ & JF & 19 & $<1$ & 237 & 1070 & 73 & 22 & 148 & 70 & 36 & 6 & 30 & 90 & 18 & 2 & $<5$ & 35 & 9 \\
\hline MI-VIII-65 & $\mathrm{HTiO}_{2}$ & JF & 28 & 4 & 253 & 1521 & 1392 & 39 & 252 & 30 & 15 & $<20$ & 10 & 120 & 24 & 2 & $<5$ & 75 & 23 \\
\hline MI-III-18 & $\mathrm{HTiO}_{2}$ & JF & 30 & 1 & 266 & 613 & 639 & 36 & 187 & $<20$ & 37 & 30 & 30 & 130 & 22 & 2 & $<5$ & 9 & 20 \\
\hline MI-BR-56 & $\mathrm{HTiO}_{2}$ & JF & 29 & 2 & 235 & 1252 & 674 & 49 & 655 & 40 & 29 & 30 & 30 & 140 & 22 & 2 & $<5$ & 16 & 31 \\
\hline
\end{tabular}


Table 1. Continuation

\begin{tabular}{|c|c|c|c|c|c|c|c|c|c|c|c|c|c|c|c|c|c|c|c|c|c|}
\hline Sample & ype & Unit & $\mathbf{L a}$ & $\mathrm{Ce}$ & Pr & Nd & Sm & Eu & Gd & $\mathbf{T b}$ & Dy & Ho & Er & $\mathbf{T m}$ & $\mathbf{Y b}$ & Lu & Hf & Ta & $\mathbf{P b}$ & th & $\mathbf{U}$ \\
\hline & $\mathrm{O}_{2}$ & BS & 6 & 1 & 17.8 & 75.1 & 15.3 & 4.42 & 12.7 & 1.8 & 9.5 & 1.8 & 4.9 & 0.65 & & 0.57 & 7 & 1.6 & 7 & .5 & 1.6 \\
\hline$-\mathrm{C}$ & $\mathrm{TiO}_{2}$ & BS & & 114 & & 65.2 & 10 & 3.77 & 11.5 & 1.7 & 9. & 1.8 & 5.3 & 76 & 4.8 & .7 & 9 & 1.5 & 6 & 0.8 & 0.2 \\
\hline RE-CE-19 & $\mathrm{HTiO}_{2}$ & BS & 28.8 & 64 & 8.58 & 36.7 & 8 & 2.57 & 7.3 & 1.1 & 6.5 & 1.3 & 3.4 & 0.49 & s & 0.43 & 3.7 & 1 & $<5$ & 2 & $<0.1$ \\
\hline$C F_{3} 20 \mathrm{R}$ & $\mathrm{HTiO}_{2}$ & BS & 67 & 18 & 10.9 & 44.9 & 9.1 & 2.08 & 78 & 1.1 & 62 & 12 & 3.1 & 0.42 & 2.5 & 0.38 & 4 & 1.3 & $<5$ & 8.8 & 0.1 \\
\hline E-CE-2A & $\mathrm{HTiO}_{2}$ & BS & 353 & 74.5 & 9.61 & 40.5 & 8.6 & 2.71 & 9 & 13 & 74 & 1.5 & 42 & 0.58 & 3.6 & 0.56 & 5.9 & 1 & 7 & 0.4 & 0.2 \\
\hline F-CF-12 & $\mathrm{HTiO}_{2}$ & BS & 5 & 130 & 17.1 & 70.8 & 14.7 & 3.66 & 13.1 & 19 & 0.8 & 2.1 & 8 & 79 & 4.9 & 72 & 3.9 & 1.5 & 7 & 0.5 & 0.2 \\
\hline RE-CE-13B & $\mathrm{HTiO}_{2}$ & BS & 3 & 77.5 & 10.1 & 428 & 9 & 261 & 91 & 1.3 & 7.7 & 1.5 & 4.4 & 06 & 3.7 & 55 & 6.1 & 1 & 6 & 6 & 0.2 \\
\hline C & $\mathrm{HTiO}_{2}$ & BS & 59.4 & 125 & 16.1 & 65.4 & 13 & 3.8 & 11.3 & 1 & 97 & 1.8 & 5.2 & .72 & 4.3 & 0.64 & 10.1 & 1.4 & 9 & 9 & 0.2 \\
\hline RE-CE-28C & $\mathrm{HTiO}_{2}$ & BS & 20.1 & 44.4 & 5.88 & 25.6 & 6.1 & 2.15 & 5.6 & 0.9 & 5.5 & 1.1 & 3.1 & 0.46 & 2.9 & 0.42 & 4.6 & 1 & $<5$ & .2 & 0.4 \\
\hline E-CE-29 & $\mathrm{HTiO}_{2}$ & BS & 40 & 84.4 & 10.5 & 42 & 8.4 & 2.83 & o & 1 & 6.9 & 1.3 & 3.8 & 53 & 3.3 & .5 & 10.1 & 1 & 6 & 8 & 0.2 \\
\hline RE-CE & $\mathrm{HTiO}_{2}$ & BS & 53 & 1 & 14. & 60.2 & 12.1 & 3.56 & 11 & 1.6 & 9 & 1.8 & 4.9 & 0.7 & 4.3 & 64 & .7 & 1.3 & 8 & 1 & 0.2 \\
\hline E-CE- & $\mathrm{HTiO}_{2}$ & BS & 37.5 & 81.7 & 10.8 & 47.2 & 10.1 & 0.1 & 9.6 & 1.6 & 8.6 & 1.6 & 4.5 & (3) & 4 & 59 & 6.2 & 12 & 5 & 0.3 & 0.1 \\
\hline RE-CE-3A & $\mathrm{LTiO}_{2}$ & BS & 24.9 & 50.6 & 6.5 & 26.5 & 55 & 1. & 51 & 0.7 & 4.3 & 0.8 & 2.3 & 0.33 & 2.1 & 0.31 & 4 & 0.6 & 6 & 0.5 & 0.1 \\
\hline RE-C & $\mathrm{LTiO}_{2}$ & BS & 18.1 & 38.8 & 5.08 & 21.5 & 4 & 1.8 & 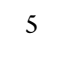 & 0.8 & 4.4 & 0 & 2.6 & 0.36 & 2. & L & 3 & .5 & $<5$ & 0.5 & 0.1 \\
\hline $\mathrm{K}$ & $\mathrm{iO}_{2}$ & BS & 24.9 & 50 & 6.53 & 27 & 5. & 1.96 & 6 & 1 & 5.9 & 1. & 3.3 & 0.46 & 3 & 4 & 4.6 & 0.7 & 8 & 2.9 & 0.8 \\
\hline $\mathrm{R}$ & $\mathrm{iO}_{2}$ & BS & 3 & 73 & 9. & 38.5 & 8 & 2.02 & 7.6 & 1. & 6.8 & 1.2 & 3.3 & 0.51 & 3 & 8 & .7 & 1.1 & 11 & .1 & 0.9 \\
\hline RE-CE-3 & $\mathrm{LTiO}_{2}$ & BS & 27.2 & 60.4 & 7.89 & 32.6 & 7.1 & 2.01 & 65 & 1 & 59 & 1.2 & 3.3 & 0.45 & 2.8 & 41 & 4 & 0.5 & $<5$ & 0.9 & 0.2 \\
\hline RE-CE-11 & $\mathrm{LTiO}_{2}$ & BS & 35.2 & 67.1 & 8.42 & 35.1 & 6.9 & 2.29 & 6.6 & 1 & 5.4 & 1.1 & 3.1 & 0.43 & 2.7 & 37 & 5.5 & .4 & 7 & 0.5 & 0.1 \\
\hline RE-CE- 1 & $\mathrm{LTiO}_{2}$ & BS & 17.2 & 35.8 & 4.69 & 20.3 & 4.2 & 1.84 & 11 & 0.7 & 3.7 & 0. & & 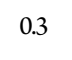 & 1.9 & 28 & 1.5 & 17 & $<5$ & 0.1 & $<0.1$ \\
\hline MI-I & $\mathrm{LTiO}_{2}$ & AND & 6.4 & 17 & 2.5 & 1 & 3.4 & 1.13 & 17 & 0.1 & 4 & 0.8 & 2.3 & 34 & 2.1 & 0.34 & 1.6 & 4 & 5 & .6 & 0.2 \\
\hline MI-B & $\mathrm{LTiO}_{2}$ & AND & 5 & 1 & 12.7 & 45.2 & 7. & 2.07 & $5-$ & 0.8 & 4.5 & 0. & 2.5 & 0.38 & 2.4 & 37 & 4.1 & 6 & 9 & 9 & 1.2 \\
\hline $\mathrm{Pf}$ & $\mathrm{LTiO}_{2}$ & AND & 18 & 38 & 4.87 & 20. & 4. & 1 & 16 & 0.8 & 4.6 & 0 & 2.6 & 0.41 & 2.5 & 38 & 6 & .9 & 5 & 3 & 0.4 \\
\hline M & $\mathrm{LTiO}_{2}$ & $\mathrm{JF}$ & 8.2 & 5 & 2.12 & & 2.1 & & 2.2 & 0.4 & 2.5 & 0. & 1.3 & ק & 1.3 & 21 & 4 & 1 & 5 & 4.4 & 0.1 \\
\hline M & $\mathrm{LTiO}_{2}$ & IF & 1 & 4.2 & 3.76 & 15.9 & 3. & 1.11 & 3.3 & 0.6 & 7 & 0.7 & & 0.31 & 2 & 28 & 0.5 & $<0.1$ & $<5$ & 0.4 & 0.2 \\
\hline SP-BR-16 & $\mathrm{LTiO}_{2}$ & $\mathrm{JF}$ & 22.3 & 16.7 & 6.05 & 25.2 & 6.3 & .99 & 6.5 & .1 & 6.6 & 1.3 & 3.5 & 15 & 3.2 & 49 & 1.9 & 3 & $<5$ & .4 & 0.1 \\
\hline MI-II-62 & $\mathrm{LTiO}_{2}$ & $\mathrm{JF}$ & 11.7 & 22.6 & 12 & 12.8 & 3.2 & 0.99 & 3.7 & 0.6 & 3.9 & 0.8 & 2.4 & 38 & 2.4 & 0.33 & 2.2 & .3 & $<5$ & 0.6 & 0.1 \\
\hline MI-V-40 & $\mathrm{LTiO}_{2}$ & $\mathrm{JF}$ & 15.3 & 33.6 & 4.41 & 18.6 & 4.3 & 1.22 & 3. & 0.6 & 3.1 & 0.6 & 1.8 & 28 & 1.8 & 5 & 2 & & 8 & 1.2 & 0.3 \\
\hline MI-BL-2? & $\mathrm{LTiO}_{2}$ & $\mathrm{JF}$ & 1 & & 2.76 & 12.1 & 2. & 0.97 & 1 & 0.5 & 3 & 0. & 1.8 & 26 & 1.7 & 44 & 1.8 & .3 & $<5$ & .8 & 0.4 \\
\hline $\mathrm{P} f$ & $\mathrm{LTiO}_{2}$ & $\mathrm{JF}$ & 31.9 & 65.5 & 8.65 & 36.9 & 8 & & & 1.1 & 6.4 & 1.2 & 3.4 & & J & 0.44 & 2.6 & & 5 & 4 & 0.2 \\
\hline קח & $\mathrm{LTiO}_{2}$ & $\Gamma$ & & 53.3 & & 3 & 6.7 & & & & & 0.8 & 2.7 & & 2.2 & & 3.1 & & 8 & 11 & 1.1 \\
\hline SP-BR-06A & $\mathrm{LTiO}_{2}$ & 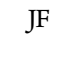 & & 104 & & 66.5 & 16 & 2.26 & 123 & 2.1 & 11.2 & & 5.4 & 0.76 & 4.5 & 68 & .1 & & 6 & 7 & 1.6 \\
\hline $\mathrm{P} f$ & $\mathrm{LTiO}_{2}$ & $\mathrm{JF}$ & 23.3 & 5 & 8.08 & 34.6 & 7. & 1.59 & 5.9 & 0.8 & 4.5 & 0.9 & 2.4 & 0.4 & 2.5 & 36 & 1 & 6 & $<5$ & 0.3 & 0.3 \\
\hline SP-BR-06 & $\mathrm{LTiO}_{2}$ & $\mathrm{JF}$ & 22 & 57.8 & 8.87 & 42.6 & 10 & 2.61 & 9.2 & 1.4 & 7.3 & 1.4 & 3.8 & 1 & 3 & 6 & 1.9 & 3 & $<5$ & 1.7 & 0.7 \\
\hline SP-BR-17B & $\mathrm{LTiO}_{2}$ & $\mathrm{JF}$ & 5.4 & 13.2 & 2.16 & 10.7 & 3.2 & .13 & 3.8 & 0.7 & 4.2 & 0.8 & 2.4 & 0.35 & 2.3 & 0.37 & 1.8 & 0.3 & $<5$ & 0.3 & $<0.1$ \\
\hline MI-BR-19C & $\mathrm{LTiO}_{2}$ & $J F$ & 24.9 & 55.9 & 6. & 25.7 & 6 & 1.77 & 5.9 & 1.1 & 6.2 & 1.2 & 3.3 & & 3.2 & & 2.2 & 1 & 7 & .1 & 0.1 \\
\hline MI-BR-1 & $\mathrm{LTiO}_{2}$ & $\mathrm{JF}$ & 5. & 1 & 2 & 99.4 & 23.1 & 2.06 & 19.1 & 3.1 & 17 & 3.2 & 8.8 & 34 & 0 & 8 & 3.1 & & 12 & 4.8 & 0.3 \\
\hline PA-BE-S & $\mathrm{LTiO}_{2}$ & $\mathrm{JF}$ & 10 & 2 & 3.22 & 13 & 2 & 1.13 & 3 & 0.6 & 3.6 & 0 & 2.4 & 39 & 2.5 & & 0.9 & 0.3 & $<5$ & 2.5 & 0.6 \\
\hline MI-BE-83A & $\mathrm{LTiO}_{2}$ & $\mathrm{JF}$ & 2 & 3 & 6.91 & 2 & 5 & 1.85 & 5.1 & 0.8 & 4.8 & 0.9 & 2.6 & s & 2.5 & 37 & 2 & 0.6 & 7 & 1.4 & 0.3 \\
\hline MI-IX-03B & $11100_{2}$ & st & 27 & 60 & 1. & 3 & 6 & 1.89 & 5.7 & 0.8 & 4.3 & 0.8 & 2.1 & 0.5 & 1.8 & 26 & . & S & $<5$ & 1 & 0.2 \\
\hline M & 2 & $\mathrm{JF}$ & 6 & 1 & 17.3 & 7 & 17 & 3.52 & 10.8 & 1.6 & 7.7 & 1.3 & 3.6 & 0.52 & 3. & 0.42 & . & 1.1 & 0 & 4.2 & 1 \\
\hline M & $\mathrm{HTiO}_{2}$ & $J F$ & 3 & 76 & 10.3 & 4 & 9.4 & 2.51 & 7.9 & 1.2 & 6.9 & 1.3 & 3.5 & 6 & 3.3 & 4 & 3.5 & 9 & $<5$ & .3 & $<0.1$ \\
\hline MI-BF & $\mathrm{HTiO}_{2}$ & $\mathrm{JF}$ & 6 & 1 & 18.8 & 76.6 & 15.2 & 3 & 12.6 & 1. & 9.6 & 1.7 & 4.8 & 0.73 & 4.5 & 0.63 & 11.8 & 1.2 & 8 & 0.9 & 0.1 \\
\hline
\end{tabular}


$\mathrm{CAB})$, indicating arc-related environments. As mafic rocks of this group crop out only within the orthogranulites of the Juiz de Fora complex, we suggest that they belong to the convergent Rhyacian history of the basement.
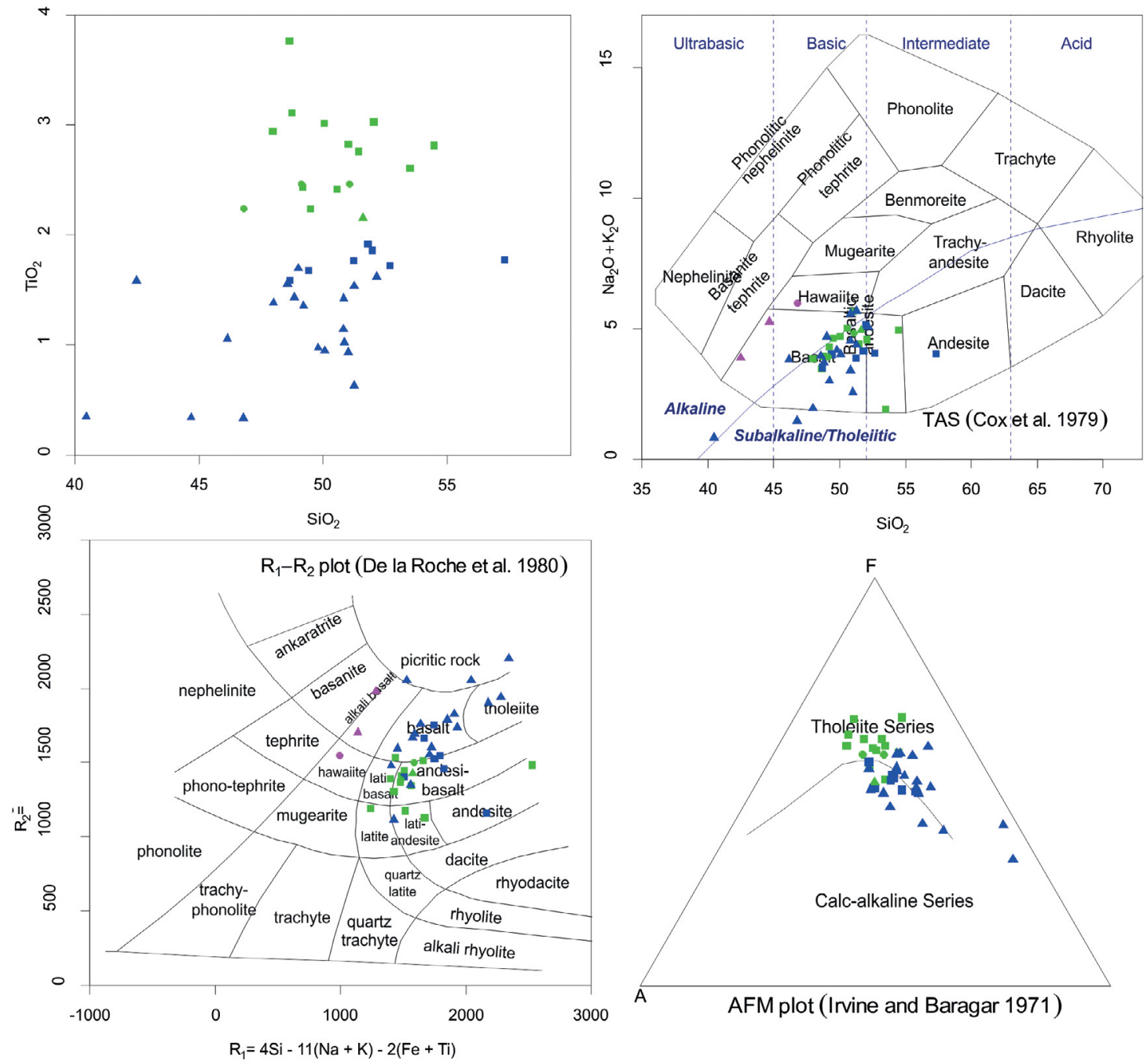

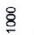

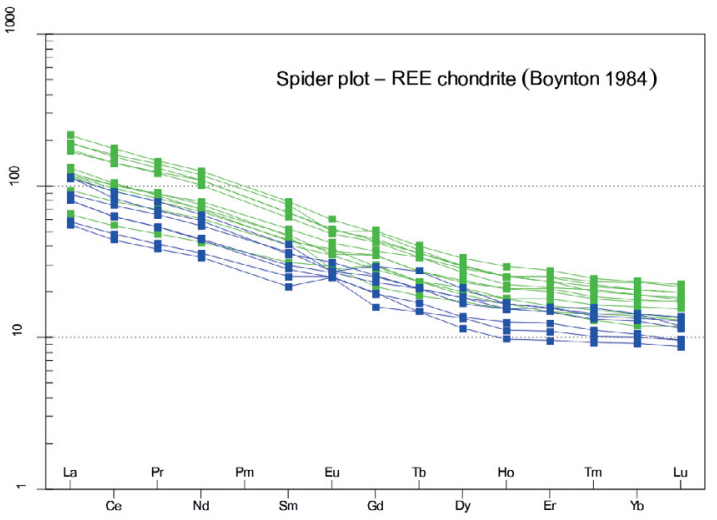

\begin{tabular}{|l|l|}
\hline Symbols & Low- $\mathrm{TiO}_{2} \mathrm{High}-\mathrm{TiO}_{2}$ Alkaline \\
Barreiro Suite & \\
Juiz de Fora enclaves & \\
Andrelândia Group enclaves &
\end{tabular}

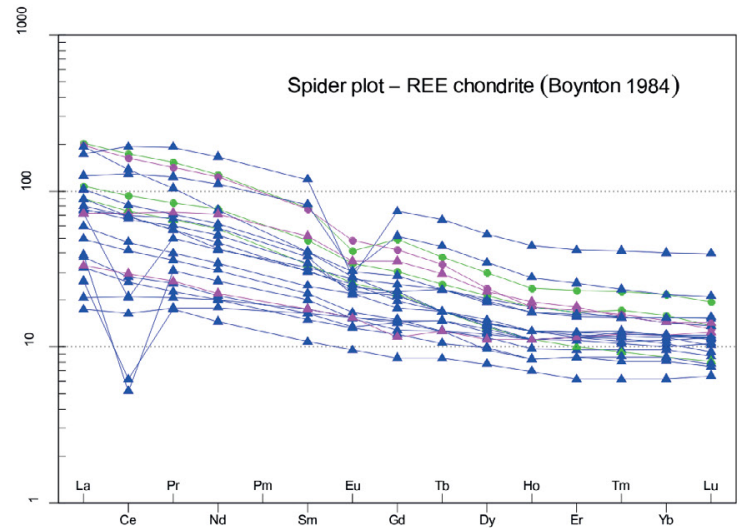

Figure 7. Geochemical data for the rocks of the Barreiro Suite and mafic enclaves within the country rocks: (A) binary $\mathrm{TiO}_{2} \times \mathrm{SiO}_{2} \mathrm{diagram}$ with subdivision into two groups; (B) chemical classification of the analysed rocks in the TAS diagram of Cox et al. (1989); (C) chemical classification in the R1 $\times$ R2 diagram of De La Roche et al. (1980); (D) AFM diagram of Irvine and Baragar (1971); (E) chrondrite-normalized spidergram of the High- $\mathrm{TiO}_{2}$ and $\mathrm{Low}-\mathrm{TiO}_{2}$ groups of the Barreiro Suite; $(\mathrm{F})$ chrondrite-normalized spidergram including alkaline basic enclaves in the Andrelândia Group and Juiz de Fora Complex. Both spidergrams were normalized using the chondrite composition of Boynton (1984). 
Enclaves of groups 1 to 3 (both Andrelândia Group and Juiz de Fora Complex) and all Barreiro Suite samples plot in the intraplate to passive margin extensional settings (Fig. 8).
On the other hand, the Group 4 of the Juiz de Fora Complex (CAB to IAT convergent tectonic settings) is probably related with the Rhyacian history of the basement (Fig. 8).
A

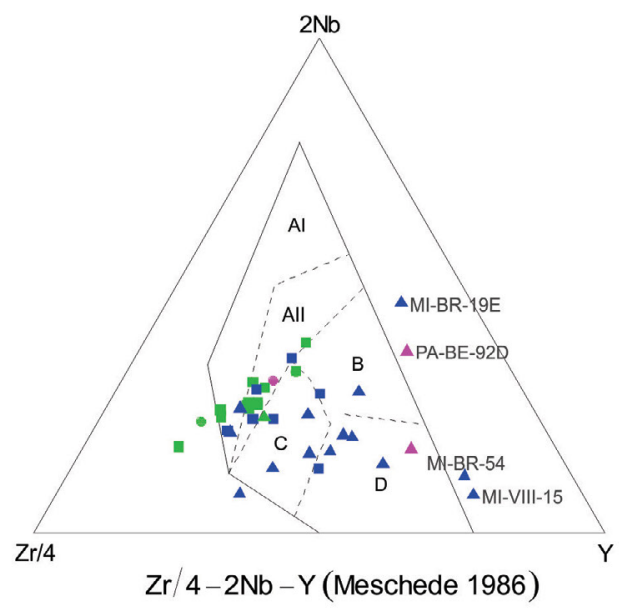

C

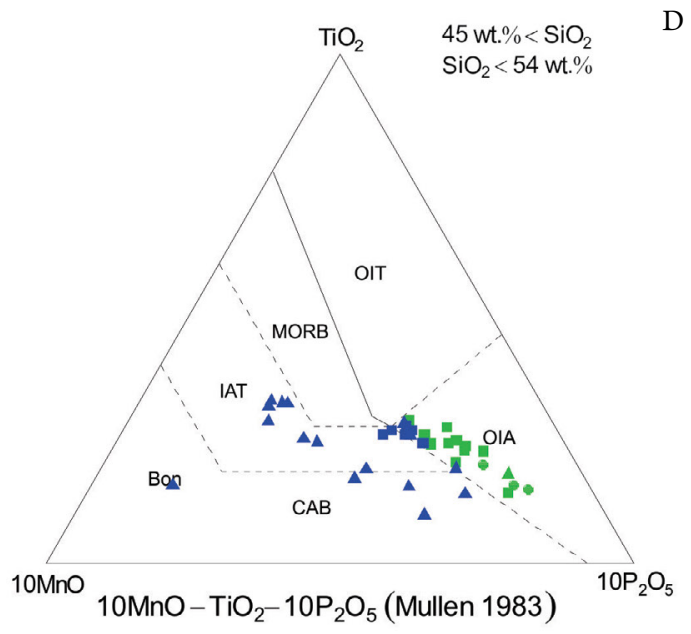

E

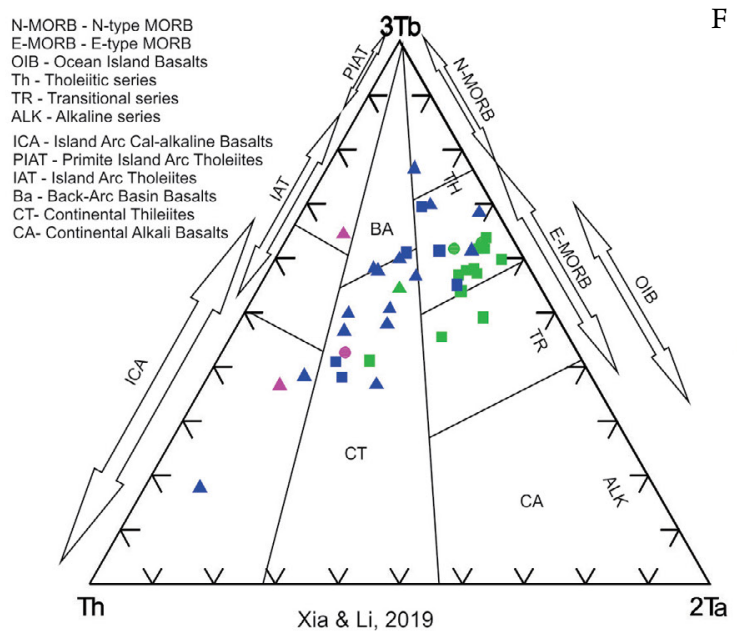

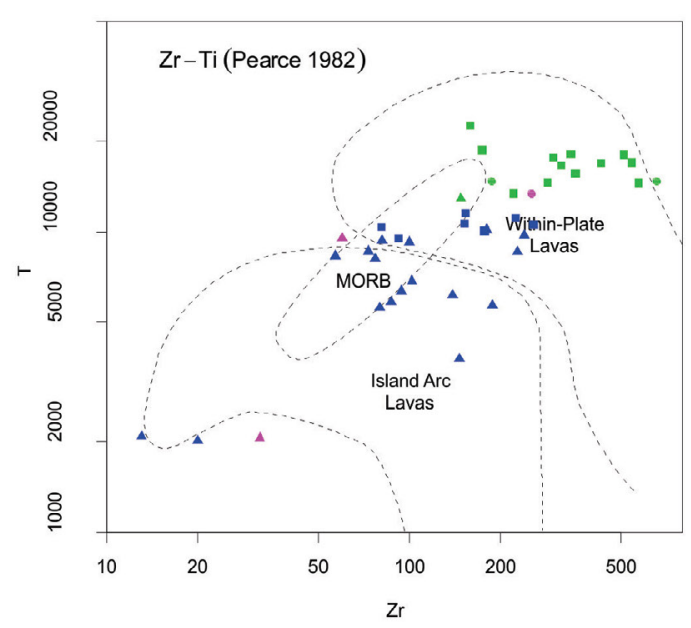
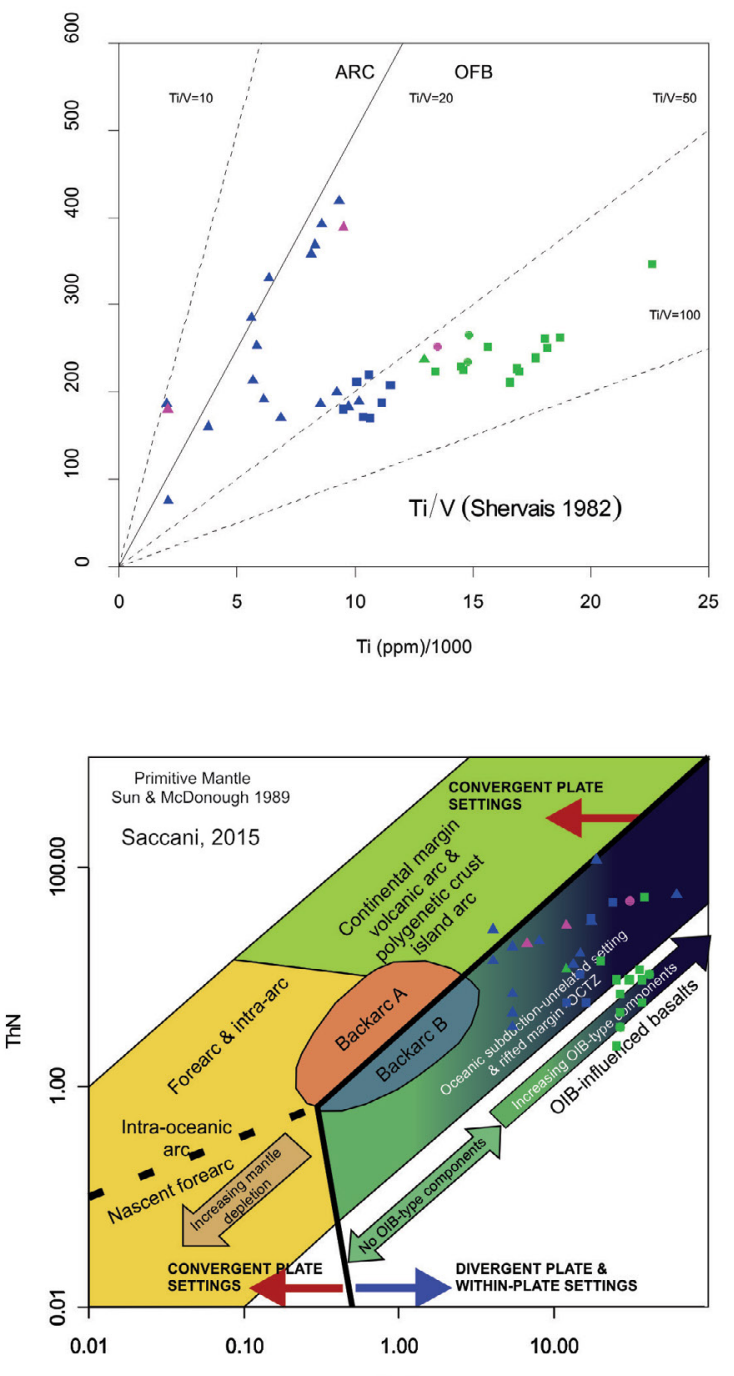

$\mathrm{NbN}$

Symbols Low- $\mathrm{TiO}_{2} \mathrm{High}-\mathrm{TiO}_{2}$ Alkaline
Barreiro Suite
Juiz de Fora enclaves
Andrelândia Group enclaves

Figure 8. Plot of analyzed sample in tectonic diagrams: (A) Zr/4- 2Nb-Y triangular diagram of Meschede (1986); (B) Zi $\times$ Ti of Pearce (1982); (C) TiO2 x $10 \mathrm{MnO} \times 10 \mathrm{P} 2 \mathrm{O} 5$ triangular diagram of Mullen (1983); and (D) Ti x V diagram of Shervais (1982), (E) triangular diagram of Cabanis and Thiéblemont (1988), in Xia and Li (2019); (F) normalized binary diagram of Scanni (2015) (normalized by Sun \& McDonough 1989) 


\section{U-PB GEOCHRONOLOGY AND HF ISOTOPES OF THE BARREIRO SUITE}

In order to constrain the age of the intraplate magmatism of the Barreiro Suite, sample MI-BR-68, a diorite, was selected for $\mathrm{U}-\mathrm{Pb}$ analysis. This sample produced the largest number of recovered zircons suitable for geochronological investigation.

\section{Preparation and analytical procedures}

The sample preparation involved crushing (ca. $30 \mathrm{~kg}$ ) and manual panning, followed by density and magnetic separation in the LGPA laboratory of the UERJ. Zircons typically show pale brown to pink colors, with short prismatic habit (2:1 to 3:1 aspect ratios), and less commonly with sub-equant shapes. Scanning electron microscope cathodoluminescence images were obtained at the MULTILAB facilities of the UERJ. Most of the grains display complex zoning patterns, rounded bright overgrowths and resorption textures that, with typical soccer ball grains, are typical of high-grade metamorphism (Rubatto 2017).

$\mathrm{U}-\mathrm{Pb}$ isotopic data were obtained at the Geochronology Laboratory of the Universidade de Brasília (UnB) using a Thermo-Finnigan Neptune multicollector inductively coupled plasma mass spectrometer connected to a "New Wave $213 \mathrm{~mm}$ Nd-YAG” laser beam with spot diameters of 30 to $50 \mu \mathrm{m}$, and frequency of 6 to $10 \mathrm{~Hz}$. Bracketing technique of analyses, described in detail by Bühn et al. (2009), involved the use of the JG1, Temora 2 and FC1 standards. For the standard and sample analyses, isotope ratio measurements consist of blocks of 40 cycles, each with $1.049 \mathrm{sec}$ duration.

Analyses with either errors higher than $10 \%$ for isotope ratios, ${ }^{204} \mathrm{~Pb}$ contents higher than $0.5 \%$, or discordance above $5 \%$, were not considered in the age calculations. Analyses with ${ }^{206} \mathrm{~Pb} /{ }^{204} \mathrm{~Pb}$ ratios higher than 3,000 indicate negligible common $\mathrm{Pb}$ contents, obviating the need for common $\mathrm{Pb}$ correction. When necessary, the correction is made with the in-house spreadsheet of Bühn et al. (2009), following Stacey and Kramers (1975). Age calculations and plots were performed with the Isoplot V.3 software of Ludwig (2003).

The Lu-Hf analyses were performed at the University of Notre Dame (USA), using a UP193 nm laser ablation system coupled to a NuPlasma II Multi-Collector ICPMS. The Lu-Hf spots were located as the same zircon texture domains of the $\mathrm{U}-\mathrm{Pb}$ spots. Analytical conditions were laser spots of $50 \mu \mathrm{m}$ diameter, $60 \mathrm{~s}$ of ablation after a run of $30 \mathrm{~s}$ for background, pulse of $5 \mathrm{~Hz}$ corresponding to an energy fluence of $\sim 10 \mathrm{~J} /$ $\mathrm{cm}^{2}$. Methodology followed the protocol of Simonetti and Neal (2010), using the Plesovice, BR266 and 1500 standards. Mass interference of ${ }^{176} \mathrm{Lu}$ with ${ }^{176} \mathrm{Hf}$ was corrected using the ${ }^{176} \mathrm{Lu} /{ }^{175} \mathrm{Lu}$ ratio of 0.02655 (International Union of Pure and Applied Chemistry 1998), and the decay constant of ${ }^{176} \mathrm{Lu}$ of $867 \times 10^{-11}$ (Söderlund et al. 2004). Chondritic ratios used for $\varepsilon_{\mathrm{Hf}}$ calculations were ${ }^{176} \mathrm{Hf} /{ }^{177} \mathrm{Hf}=279718,{ }^{176} \mathrm{Lu} /{ }^{177} \mathrm{Hf}=$ 0.0336 (Bouvier et al. 2008), and present-day depleted manthe ratios of ${ }^{176} \mathrm{Hf} /{ }^{177} \mathrm{Hf}=0.28325$ and ${ }^{176} \mathrm{Lu} /{ }^{177} \mathrm{Hf}=0.0388$ (Griffin et al. 2000; updated by Andersen et al. 2009).

\section{$\mathrm{U}-\mathrm{Pb}$ results}

Analysis of sample MI-BR-68 produced 45 spots (Tab. 2), nine of which were discarded due to poor analytical parameters such as isotope ratios errors higher than $10 \%,{ }^{204} \mathrm{~Pb}$ contents higher than $0.5 \%$, or discordance above $5 \%$.

All zircons plot along the Concordia between ca. $780 \mathrm{Ma}$ and ca. $610 \mathrm{Ma}$ (Fig. 9), perhaps suggesting ion diffusion under high-temperatures of the regional granulite facies metamorphism. It also confuses distinction between igneous and metamorphic zirons on the basis of zircon textures and Th/U (Rubatto 2017). However, based on the bimodal pattern of the probability density plot of $207 \mathrm{~Pb} / 206 \mathrm{~Pb}$ ages (Fig. 9), a group of 8 zircons with concentric oscillatory zoning and relatively high Th/U ratios (Rubatto 2017) yielded a Concordia age of $766 \pm 13 \mathrm{Ma}$, interpreted as the age of reference for the mafic magmatism. The cluster of younger zircons, with relatively lower $\mathrm{Th} / \mathrm{U}$ ratios, yielded a Concordia age of $619 \pm$ $6 \mathrm{Ma}$, interpreted as the best estimate for the age of the metamorphic overprint. This latter age is comparable with the early metamorphic ages reported for the Ribeira Belt (Bento dos Santos et al. 2007, Machado et al. 1996, Heilbron \& Machado 2003). Field observations support this interpretation, such as the presence of thin leucocratic intrusive veins and partial digestion of the mafic enclaves of the Barreiro Suite within the syn-collisional granitoid rocks.

Three inherited zircons, with ages of ca. $1.38 \mathrm{Ga}, 2.14 \mathrm{Ga}$ and $2.15 \mathrm{Ga}$, reflect the basement associations of the Occidental terrane of the Ribeira Belt, confirming the intraplate setting of the Barreiro Suite as indicated by geochemical criteria.

\section{Lu-Hf results}

Lu-Hf analyses (Tab. 3) were performed on the same sample as the U-Pb analyses. Zircon grains were selected for the investigation of crystallization age, metamorphic overprint and inheritance. Measured ${ }^{176} \mathrm{Hf} /{ }^{177} \mathrm{Hf}$ ratios vary between 0.281927 and 0.282559 , while ${ }^{176} \mathrm{Lu} /{ }^{177} \mathrm{Hf}$ ratios are below 0.0015 .

Zircons with late Tonian ages, interpreted as crystallization ages, yield a broad spectrum of $\mathrm{T}_{\mathrm{DM}} \mathrm{Hf}$ model ages, as expected for intraplate basaltic rocks, ranging from ca. 1.75 to $0.96 \mathrm{Ga}$ with predominantly positive $\varepsilon \mathrm{Hf}_{(\mathrm{t}=766)}$ values between +5.61 and +0.24 . Two grains yielded negatives $\varepsilon \mathrm{Hf}_{(\mathrm{t}=766)}$ values of -10.97 and of -4.07 (Fig. 10).

On the other hand, most of the Ediacaran metamorphic zircons and overgrowths, with U-Pb ages between 624 and $594 \mathrm{Ma}$, yielded $\mathrm{Hf}_{\mathrm{DM}}$ model ages between $1.80 \mathrm{Ga}$ and $1.04 \mathrm{Ga}$, with negative $\varepsilon \mathrm{Hf}_{(\mathrm{t}=766)}$ values between -10.86 and -1.82 . The metamorphic zircons are interpreted as having grown from in situ melting films within the basic rocks, the preservation of primary ${ }^{176} \mathrm{Hf} /{ }^{177} \mathrm{Hf}$ compositions in metamorphic zircon can be usually attributed to subsolidus recrystallization (Zeh et al. 2010), as strongly suggested by very complex textures detected on the cathodoluminescence images. Tedeschi et al. (2018) obtained similar results for high-temperature granulites in the Southern Brasília belt.

The single grain with age of ca. $1.39 \mathrm{Ga}$ represents inheritance, with a $\mathrm{Hf} \mathrm{T}_{\mathrm{DM}}$ model age of $1.62 \mathrm{Ga}$ with $\varepsilon \mathrm{Hf}_{(\mathrm{t}=1.62)}$ of +5.61 , suggesting juvenile sources. 
Table 2. Summary of U-Pb zircon data obtained by inductively coupled plasma mass spectrometry (LA-ICPMS) (Mi-BR-68) - ZIRCON

\begin{tabular}{|c|c|c|c|c|c|c|c|c|c|c|c|c|c|c|c|c|c|c|c|}
\hline \multirow{2}{*}{ Spot/ } & \multirow{2}{*}{$\begin{array}{c}\text { Th } \\
\text { ppm p }\end{array}$} & \multirow{2}{*}{\multicolumn{2}{|c|}{$\begin{array}{c}\text { U Pb } \\
\text { ppm ppm }\end{array}$}} & \multirow{2}{*}{ Th/U } & \multirow{2}{*}{$f^{-206}$} & \multicolumn{7}{|c|}{ Ratios } & \multicolumn{6}{|c|}{ Age (Ma) } & \multirow{2}{*}{$\begin{array}{c}\text { Discordance } \\
\%\end{array}$} \\
\hline & & & & & & ${ }^{207} \mathbf{P b}^{* / 235} \mathbf{U}$ & $1 \sigma$ & ${ }^{206} \mathbf{P b}^{*} /{ }^{238} \mathbf{U}$ & $1 \sigma$ & Rho & ${ }^{207} \mathbf{P b}^{*} /{ }^{206} \mathbf{P b}^{*}$ & $1 \sigma$ & ${ }^{206} \mathrm{~Pb} /{ }^{238} \mathrm{U}$ & $1 \sigma$ & ${ }^{207} \mathbf{P b} /{ }^{235} \mathrm{U}$ & $1 \sigma$ & ${ }^{207} \mathrm{~Pb} /{ }^{206} \mathrm{~Pb}$ & $1 \sigma$ & \\
\hline Z-01 & 176 & 360 & 41 & 0.49 & 0.0009 & 0.81869 & 3.63 & 0.09884 & 3.10 & 0.85 & 0.06008 & 1.89 & 608 & 19 & 607 & 22 & 606 & 11 & 0 \\
\hline Z-02 & 132 & 96 & 14 & 1.38 & 0.0031 & 1.03905 & 4.70 & 0.12048 & 2.83 & 0.60 & 0.06255 & 3.75 & 733 & 21 & 723 & 34 & 693 & 26 & -6 \\
\hline Z-03 & 71 & 298 & 41 & 0.24 & 0.0007 & 1.17151 & 2.42 & 0.13013 & 1.77 & 0.73 & 0.06529 & 1.66 & 789 & 14 & 787 & 19 & 784 & 13 & -1 \\
\hline Z-04 & 150 & 350 & 39 & 0.43 & 0.0010 & 0.88157 & 2.65 & 0.10567 & 1.98 & 0.75 & 0.06051 & 1.77 & 648 & 13 & 642 & 17 & 622 & 11 & -4 \\
\hline Z-05 & 57 & 77 & 10 & 0.75 & 0.0027 & 0.81597 & 5.96 & 0.09855 & 4.61 & 0.77 & 0.06005 & 3.77 & 606 & 28 & 606 & 36 & 605 & 23 & 0 \\
\hline Z-06 & 200 & 346 & 38 & 0.58 & 0.0007 & 0.81093 & 3.33 & 0.09780 & 2.82 & 0.85 & 0.06014 & 1.77 & 602 & 17 & 603 & 20 & 608 & 11 & 1 \\
\hline Z-07 & 12 & 66 & 17 & 0.18 & 0.0019 & 2.91376 & 2.67 & 0.23946 & 2.16 & 0.81 & 0.08825 & 1.56 & 1384 & 30 & 1385 & 37 & 1388 & 22 & 0 \\
\hline Z-08 & 45 & 333 & 44 & 0.14 & 0.0006 & 1.17653 & 2.83 & 0.13035 & 2.33 & 0.82 & 0.06546 & 1.60 & 790 & 18 & 790 & 22 & 789 & 13 & 0 \\
\hline Z-09 & 41 & 687 & 70 & 0.06 & 0.0005 & 0.79912 & 3.71 & 0.09649 & 3.35 & 0.90 & 0.06007 & 1.58 & 594 & 20 & 596 & 22 & 606 & 10 & 2 \\
\hline Z-10 & 66 & 155 & 18 & 0.43 & 0.0018 & 0.85099 & 4.27 & 0.10161 & 3.17 & 0.74 & 0.06074 & 2.86 & 624 & 20 & 625 & 27 & 630 & 18 & 1 \\
\hline Z-11 & 22 & 1005 & 96 & 0.02 & 0.0003 & 0.78038 & 3.72 & 0.09518 & 3.34 & 0.90 & 0.05947 & 1.64 & 586 & 20 & 586 & 22 & 584 & 10 & 0 \\
\hline Z-12 & 26 & 168 & 21 & 0.16 & 0.0012 & 1.07514 & 3.36 & 0.12121 & 2.42 & 0.72 & 0.06433 & 2.33 & 738 & 18 & 741 & 25 & 753 & 18 & 2 \\
\hline Z-13 & 25 & 40 & 8 & 0.63 & 0.0026 & 0.84299 & 6.07 & 0.10107 & 4.51 & 0.74 & 0.06049 & 4.06 & 621 & 28 & 621 & 38 & 621 & 25 & 0 \\
\hline Z-14 & 30 & 46 & 8 & 0.65 & 0.0048 & 1.18654 & 6.45 & 0.13132 & 4.74 & 0.73 & 0.06553 & 4.38 & 795 & 38 & 794 & 51 & 791 & 35 & 0 \\
\hline Z-15 & 140 & 217 & 24 & 0.65 & 0.0012 & 0.80498 & 3.64 & 0.09731 & 2.91 & 0.80 & 0.06000 & 2.18 & 599 & 17 & 600 & 22 & 603 & 13 & 1 \\
\hline Z-16 & 52 & 276 & 30 & 0.19 & 0.0010 & 0.81484 & 4.23 & 0.09851 & 3.84 & 0.91 & 0.05999 & 1.77 & 606 & 23 & 605 & 26 & 603 & 11 & 0 \\
\hline Z-17 & 79 & 351 & 38 & 0.23 & 0.0010 & 0.81989 & 3.67 & 0.09884 & 3.24 & 0.88 & 0.06016 & 1.73 & 608 & 20 & 608 & 22 & 609 & 11 & 0 \\
\hline Z-18 & 14 & 331 & 32 & 0.04 & 0.0015 & 0.82274 & 3.71 & 0.09891 & 3.24 & 0.87 & 0.06033 & 1.80 & 608 & 20 & 610 & 23 & 615 & 11 & 1 \\
\hline Z-19 & 39 & 69 & 8 & 0.58 & 0.0025 & 0.82829 & 7.43 & 0.09977 & 5.68 & 0.76 & 0.06021 & 4.78 & 613 & 35 & 613 & 45 & 611 & 29 & 0 \\
\hline Z-20 & 82 & 62 & 9 & 1.33 & 0.0044 & 1.16436 & 5.89 & 0.12907 & 4.55 & 0.77 & 0.06543 & 3.75 & 783 & 36 & 784 & 46 & 788 & 30 & 1 \\
\hline $\mathrm{Z}-21$ & 67 & 455 & 42 & 0.15 & 0.0009 & 0.91080 & 2.90 & 0.10738 & 2.25 & 0.78 & 0.06152 & 1.83 & 658 & 15 & 657 & 19 & 657 & 12 & 0 \\
\hline Z-22 & 56 & 173 & 16 & 0.33 & 0.0023 & 0.81295 & 4.73 & 0.09820 & 3.68 & 0.78 & 0.06004 & 2.96 & 604 & 22 & 604 & 29 & 605 & 18 & 0 \\
\hline Z-23 & 45 & 297 & 26 & 0.15 & 0.0012 & 0.81363 & 3.83 & 0.09815 & 3.04 & 0.79 & 0.06013 & 2.33 & 604 & 18 & 604 & 23 & 608 & 14 & 1 \\
\hline Z-24 & 51 & 92 & 12 & 0.55 & 0.0025 & 1.03636 & 7.44 & 0.11878 & 6.17 & 0.83 & 0.06328 & 4.15 & 724 & 45 & 722 & 54 & 718 & 30 & -1 \\
\hline Z-25 & 2 & 28 & 2 & 0.08 & 0.0119 & 0.72127 & 19.74 & 0.08933 & 14.3 & 0.72 & 0.05856 & 13.7 & 552 & 79 & 551 & 109 & 551 & 75 & $\mathbf{0}$ \\
\hline Z-26 & 151 & 183 & 70 & 0.83 & 0.0004 & 7.44485 & 1.85 & 0.39657 & 1.39 & 0.76 & 0.13616 & 1.21 & 2153 & 30 & 2166 & 40 & 2179 & 26 & 1 \\
\hline Z-27 & 9 & 17 & 6 & 0.51 & 0.0023 & 7.24563 & 6.67 & 0.39303 & 4.15 & 0.62 & 0.13370 & 5.23 & 2137 & 89 & 2142 & 143 & 2147 & 112 & 0 \\
\hline Z-28 & 44 & 156 & 15 & 0.29 & 0.0024 & 0.94416 & 4.48 & 0.11023 & 3.69 & 0.82 & 0.06212 & 2.55 & 674 & 25 & 675 & 30 & 678 & 17 & 1 \\
\hline Z-29 & 69 & 115 & 12 & 0.60 & 0.0026 & 0.89659 & 5.08 & 0.10611 & 3.70 & 0.73 & 0.06128 & 3.49 & 650 & 24 & 650 & 33 & 649 & 23 & 0 \\
\hline Z-30 & 44 & 338 & 30 & 0.13 & 0.0009 & 0.81589 & 3.90 & 0.09824 & 3.41 & 0.88 & 0.06023 & 1.89 & 604 & 21 & 606 & 24 & 612 & 12 & 1 \\
\hline Z-31 & 29 & 121 & 9 & 0.25 & 0.0016 & 0.84257 & 5.18 & 0.10108 & 3.44 & 0.66 & 0.06046 & 3.87 & 621 & 21 & 621 & 32 & 620 & 24 & 0 \\
\hline Z-32 & 21 & 99 & 9 & 0.22 & 0.0024 & 1.08145 & 5.02 & 0.12212 & 3.27 & 0.65 & 0.06423 & 3.81 & 743 & 24 & 744 & 37 & 749 & 29 & 1 \\
\hline Z-33 & 60 & 166 & 14 & 0.36 & 0.0018 & 0.83414 & 4.90 & 0.10011 & 4.31 & 0.88 & 0.06043 & 2.33 & 615 & 26 & 616 & 30 & 619 & 14 & 1 \\
\hline Z-34 & 45 & 241 & 20 & 0.19 & 0.0008 & 0.84907 & 4.00 & 0.10167 & 3.26 & 0.82 & 0.06057 & 2.31 & 624 & 20 & 624 & 25 & 624 & 14 & 0 \\
\hline Z-35 & 57 & 263 & 24 & 0.22 & 0.0014 & 0.83449 & 3.34 & 0.10021 & 2.76 & 0.83 & 0.06039 & 1.88 & 616 & 17 & 616 & 21 & 618 & 12 & 0 \\
\hline Z-36 & 7 & 627 & 51 & 0.01 & 0.0005 & 0.83278 & 3.30 & 0.10023 & 2.81 & 0.85 & 0.06026 & 1.72 & 616 & 17 & 615 & 20 & 613 & 11 & 0 \\
\hline Z-37 & 29 & 87 & 8 & 0.34 & 0.0030 & 0.83633 & 6.24 & 0.10043 & 5.03 & 0.81 & 0.06040 & 3.69 & 617 & 31 & 617 & 38 & 618 & 23 & 0 \\
\hline Z-38 & 262 & 202 & 28 & 1.30 & 0.0013 & 0.92605 & 3.73 & 0.10856 & 3.04 & 0.81 & 0.06187 & 2.17 & 664 & 20 & 666 & 25 & 670 & 15 & 1 \\
\hline Z-39 & 79 & 140 & 16 & 0.57 & 0.0018 & 0.82444 & 4.94 & 0.09918 & 4.02 & 0.81 & 0.06029 & 2.87 & 610 & 24 & 611 & 30 & 614 & 18 & 1 \\
\hline $\mathrm{Z}-40$ & 60 & 178 & 19 & 0.34 & 0.0021 & 0.82405 & 3.89 & 0.09920 & 2.92 & 0.75 & 0.06025 & 2.56 & 610 & 18 & 610 & 24 & 613 & 16 & 0 \\
\hline Z-41 & 19 & 95 & 11 & 0.21 & 0.0032 & 0.87459 & 5.18 & 0.10374 & 4.08 & 0.79 & 0.06114 & 3.19 & 636 & 26 & 638 & 33 & 644 & 21 & 1 \\
\hline Z-42 & 180 & 326 & 31 & 0.56 & 0.0008 & 0.83788 & 2.81 & 0.10108 & 2.38 & 0.85 & 0.06012 & 1.50 & 621 & 15 & 618 & 17 & 608 & 9 & -2 \\
\hline Z-43 & 44 & 138 & 13 & 0.32 & 0.0018 & 0.83850 & 4.82 & 0.10095 & 3.57 & 0.74 & 0.06024 & 3.24 & 620 & 22 & 618 & 30 & 612 & 20 & -1 \\
\hline Z-44 & 369 & 312 & 39 & 1.19 & 0.0011 & 0.83308 & 2.67 & 0.09968 & 2.24 & 0.84 & 0.06062 & 1.45 & 613 & 14 & 615 & 16 & 626 & 9 & 2 \\
\hline Z-45 & 236 & 542 & 56 & 0.44 & 0.0009 & 0.87582 & 2.55 & 0.10566 & 2.07 & 0.81 & 0.06012 & 1.48 & 647 & 13 & 639 & 16 & 608 & 9 & -7 \\
\hline
\end{tabular}

${ }^{1}$ Sample and standard are corrected after $\mathrm{Pb}$ and $\mathrm{Hg}$ blanks; ${ }^{2207} \mathrm{~Pb} /{ }^{206} \mathrm{~Pb}$ and ${ }^{206} \mathrm{~Pb} /{ }^{238} \mathrm{U}$ are corrected after common $\mathrm{Pb}$ presence. $\mathrm{Common} \mathrm{Pb}$ assuming ${ }^{206} \mathrm{~Pb} /{ }^{238} \mathrm{U}-{ }^{207} \mathrm{~Pb} /{ }^{235} \mathrm{U}$ concordant age; ${ }^{3} f-206$ denotes the percentage of ${ }^{206} \mathrm{~Pb}$ that is common $\mathrm{Pb} ;{ }^{4235} \mathrm{U}=1 / 137.88^{*} \mathrm{Utotal} ;{ }^{5} \mathrm{Standard} \mathrm{GJ}-1 ;{ }^{6} \mathrm{Th} / \mathrm{U}={ }^{232} \mathrm{Th} /{ }^{238} \mathrm{U}$ ${ }^{*} 0.992743 ;{ }^{7}$ All errors in the table are calculated 1 sigma (\% for isotope ratios, absolute for ages) samples marked in bold letters are discarded. 
A

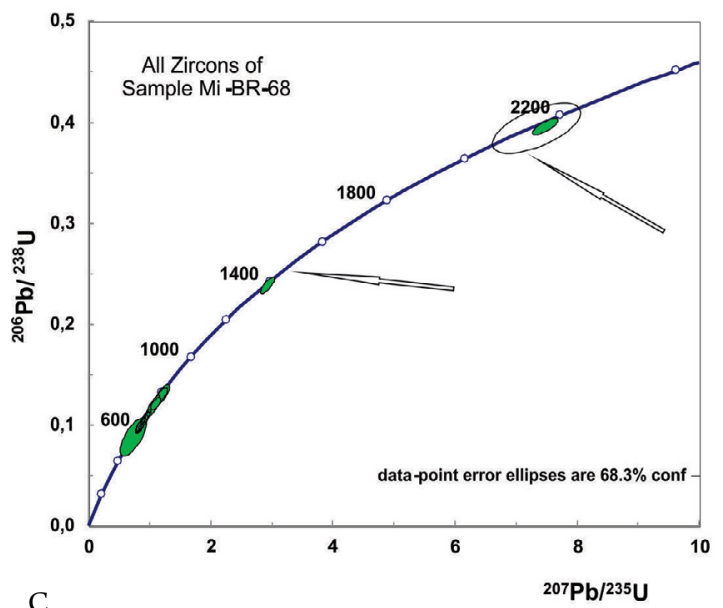

$\mathrm{C}$

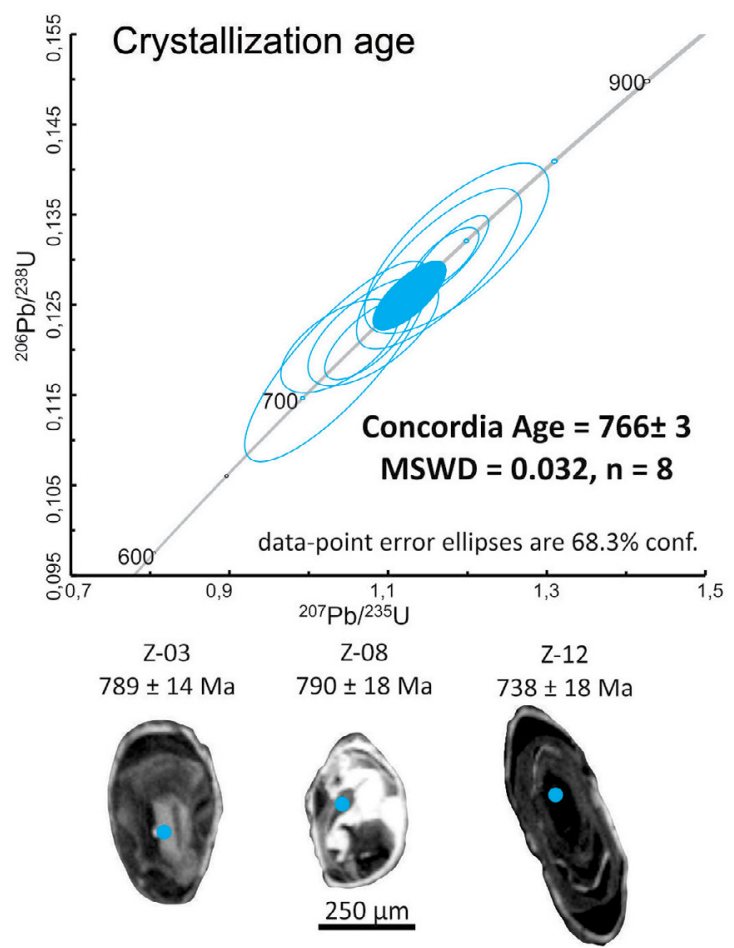

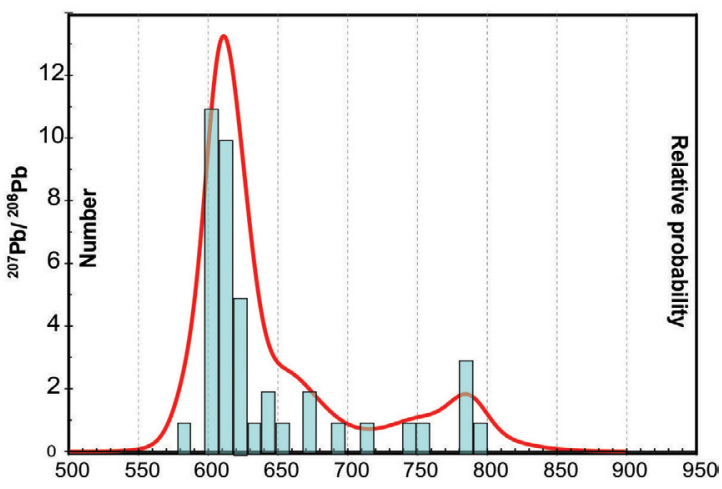

$\mathrm{D}$

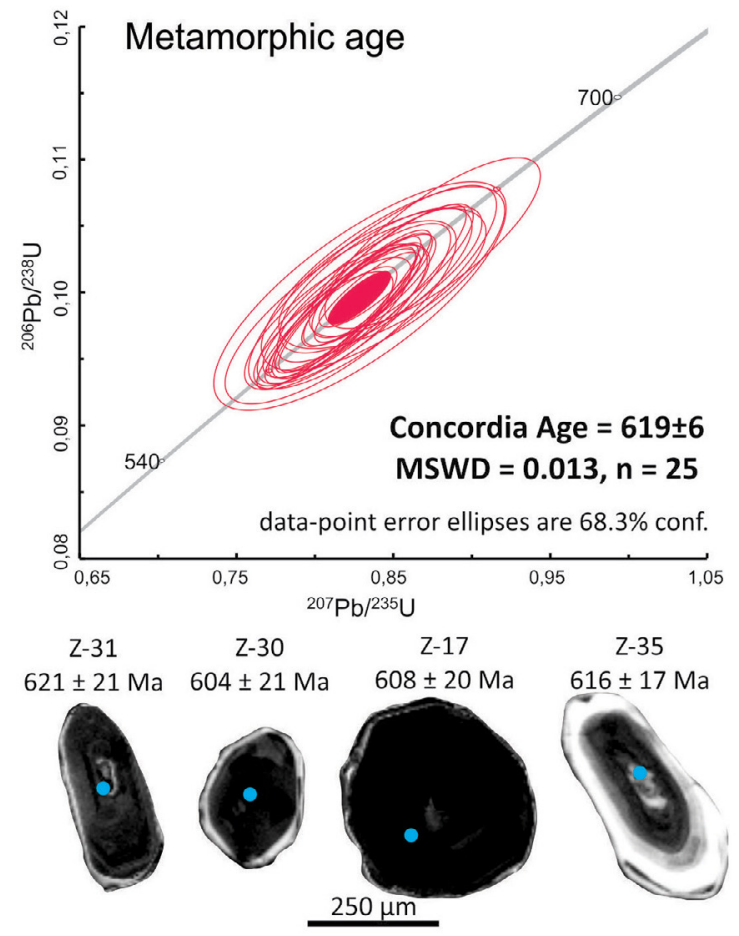

Figure 9. LA_ICPMS U-Pb data for sample MI-BR-68 of the Barreiro Suite. (A) Concordia diagram for all analysed zircons, indicating the Mesoproterozoic and Paleoproterozoic inheritance; (B) relative probability plot of ${ }^{207} \mathrm{~Pb} /{ }^{206} \mathrm{~Pb}$ ages showing the bimodal pattern; (C) calculated crystallization age of $766 \pm 3$ Ma with selected images of analysed zircons; (D) calculated high-grade metamorphic overprint age of $619 \pm 6 \mathrm{Ma}$.

Table 3. Lu-Hf data of sample MI-BR-68

\begin{tabular}{|c|c|c|c|c|c|c|c|c|}
\hline \multirow{2}{*}{ Sample } & \multirow{2}{*}{$\begin{array}{c}\text { U-Pb age } \\
(\mathrm{Ma})\end{array}$} & \multicolumn{3}{|c|}{ Sample (present-day ratios) } & \multicolumn{3}{|c|}{ Sample (initial ratios) } & \multirow{2}{*}{$\begin{array}{l}\text { T DM } \\
(\text { Ma) }\end{array}$} \\
\hline & & ${ }^{176} \mathrm{Hf} /{ }^{177} \mathrm{Hf}$ & $\pm 2 S E$ & ${ }^{176} \mathrm{Lu} /{ }^{177} \mathrm{Hf}$ & ${ }^{176} \mathrm{Hf} /{ }^{177} \mathrm{Hf}(\mathrm{t})$ & eHf $(t)$ & $\pm 2 S E$ & \\
\hline MI-BR-68_01(z36) & 616 & 0,281927 & 0,000018 & 0,000018 & 0,281927 & $-16,62$ & 0,47 & 1796 \\
\hline MI-BR-68_02(z37) & 617 & 0,282125 & 0,000021 & 0,000165 & 0,282123 & $-9,66$ & 0,49 & 1538 \\
\hline MI-BR-68_03(z16) & 606 & 0,282354 & 0,000014 & 0,000237 & 0,282351 & $-1,83$ & 0,07 & 1230 \\
\hline MI-BR-68_04(z19) & 613 & 0,282402 & 0,000013 & 0,000499 & 0,282397 & $-0,06$ & 0,00 & 1172 \\
\hline MI-BR-68_05(z17) & 608 & 0,282294 & 0,000014 & 0,000028 & 0,282294 & $-3,82$ & 0,12 & 1305 \\
\hline MI-BR-68_06(z03) & 789 & 0,282328 & 0,000014 & 0,001451 & 0,282306 & 0,69 & 0,01 & 1307 \\
\hline MI-BR-68_07(z20) & 783 & 0,282338 & 0,000016 & 0,001000 & 0,282323 & 1,17 & 0,05 & 1277 \\
\hline MI-BR-68_08(z14) & 795 & 0,281983 & 0,000015 & 0,000702 & 0,281972 & $-10,98$ & 0,52 & 1752 \\
\hline MI-BR-68_09(z12) & 738 & 0,282218 & 0,000014 & 0,000977 & 0,282204 & $-4,08$ & 0,10 & 1442 \\
\hline MI-BR-68_10(z15) & 599 & 0,282497 & 0,000015 & 0,000411 & 0,282493 & 3,02 & 0,09 & 1040 \\
\hline MI-BR-68_11(z10) & 624 & 0,282496 & 0,000014 & 0,000389 & 0,282491 & 3,53 & 0,11 & 1042 \\
\hline MI-BR-68_12(z07) & 1388 & 0,282066 & 0,000014 & 0,000165 & 0,282061 & 5,62 & 0,09 & 1617 \\
\hline MI-BR-68_15(z28) & 733 & 0,282558 & 0,000018 & 0,000664 & 0,282548 & 8,02 & 0,23 & 964 \\
\hline MI-BR-68_16(z42) & 621 & 0,282335 & 0,000015 & 0,000108 & 0,282334 & $-2,10$ & 0,05 & 1252 \\
\hline MI-BR-68_18(z22) & 604 & 0,282425 & 0,000017 & 0,000332 & 0,282422 & 0,62 & 0,02 & 1136 \\
\hline MI-BR-68_21(z09) & 594 & 0,282105 & 0,000013 & 0,000090 & 0,282103 & $-10,86$ & 0,37 & 1562 \\
\hline MI-BR-68_22(z31) & 621 & 0,282146 & 0,000015 & 0,000053 & 0,282146 & $-8,77$ & 0,30 & 1504 \\
\hline
\end{tabular}




\section{Sm-Nd AND Sr ISOTOPES}

\section{Analytical procedures}

Thirteen samples of the Barreiro suite and from mafic enclaves within Juiz de Fora Complex and Andrelândia Group country rocks were selected. Samples preparation involved fragmentation with a jaw crusher and milling at the LGPA-UERJ.

$\mathrm{Sm}-\mathrm{Nd}$ and $\mathrm{Sr}$ isotopic data were obtained by at the Laboratory Geochronology and Radiogenic Isotopes (LAGIR) of the UERJ, where chemical procedures are carried out in clean rooms using purification of Milli- $\mathrm{Q}^{\circledR}$ water and PA Merck ${ }^{\circledR}$ acids purified by repeated sub-boiling distillation. Between 25 and $50 \mathrm{mg}$ of pulverized rock samples were subjected to digestion in Savillex ${ }^{\circledR}$ vessels on a hot plate, after the addition of proportional amounts of a double ${ }^{149} \mathrm{Sm}^{-150} \mathrm{Nd}$ tracer, using a mixture of concentrated $\mathrm{HF}$ and $\mathrm{HNO}_{3} 6 \mathrm{~N}$ for 3 days, followed by further digestion with $\mathrm{HCl} 6 \mathrm{~N}$ for 2 days. Separation of $\mathrm{Sr}$ and REE used cation exchange following conventional techniques with Teflon columns filled with Biorad AG50W-X8 resin (100-200 mesh) in $\mathrm{HCl}$ medium. For the separation of Sm and Nd from the other REE, a secondary column was used with the Eichrom LN-B-25S (50-100 $\mu \mathrm{m})$ resin.

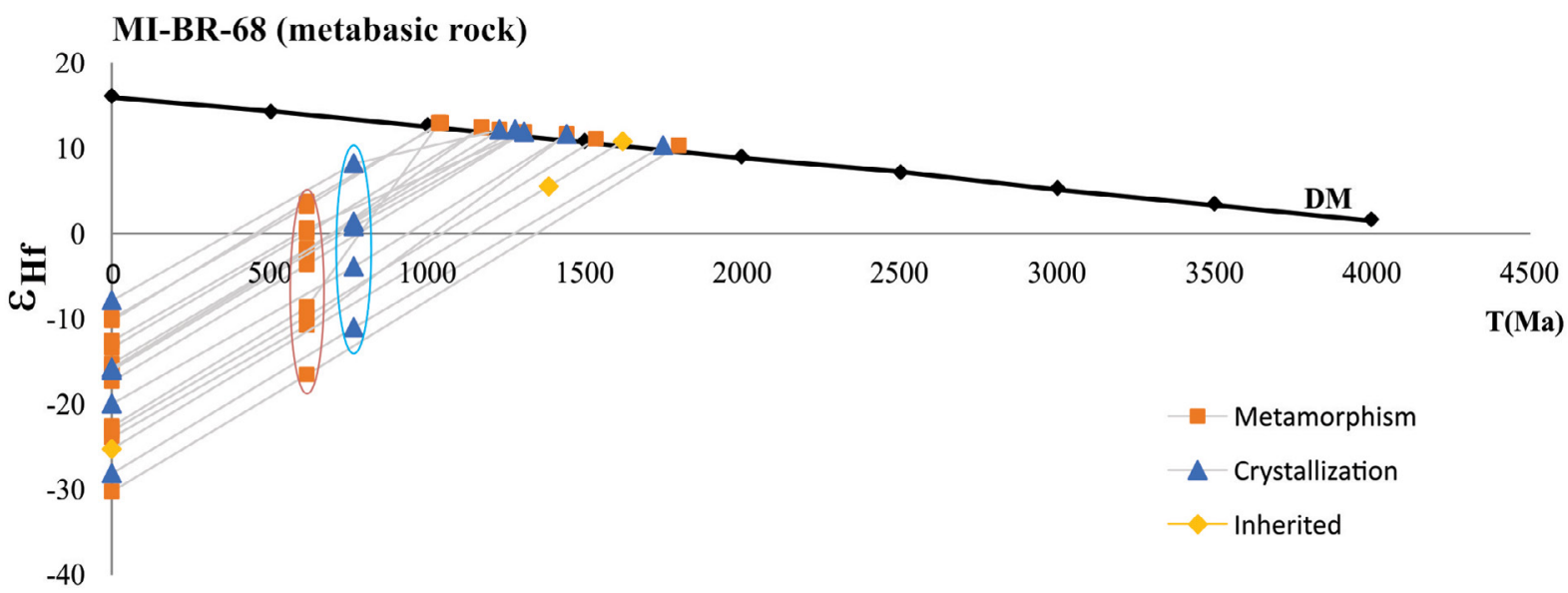

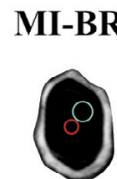

$\begin{array}{cc}594 \mathrm{Ma}(\mathrm{z} 09) & 599 \mathrm{Ma}(\mathrm{z} 15) \\ \mathrm{Th} / \mathrm{U}=0.06 & \mathrm{Th} / \mathrm{U}=0.65\end{array}$

$\varepsilon \mathrm{Hf}(\mathrm{t}) \quad \varepsilon \mathrm{Hf}(\mathrm{t})$

$-10.86$

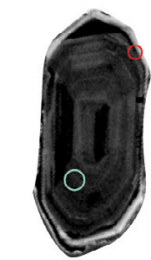

$617 \mathrm{Ma} \mathrm{(z37)}$

$\mathrm{Th} / \mathrm{U}=0.34$
$\varepsilon \mathrm{Hf}(\mathrm{t})$

$\varepsilon H f(t)$
-9.66

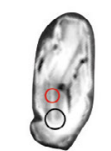

$733 \mathrm{Ma}(702)$

$\mathrm{Th} / \mathrm{U}=1.38$

$\varepsilon \mathrm{Hf}(\mathrm{t})$

$+8.02$
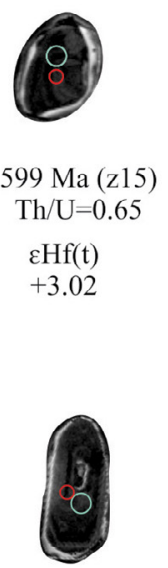

$621 \mathrm{Ma}(\mathrm{z} 31)$ $\mathrm{Th} / \mathrm{U}=0.25$ $\varepsilon \mathrm{Hf}(\mathrm{t})$ $-8.77$

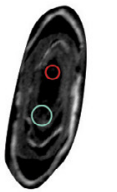

$738 \mathrm{Ma} \mathrm{(z12)}$

$\mathrm{Th} / \mathrm{U}=0.16$

$\varepsilon \mathrm{Hf}(\mathrm{t})$ $-4.08$

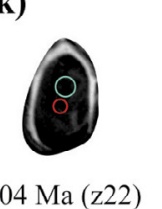

$604 \mathrm{Ma}(\mathrm{z} 22)$ $\mathrm{Th} / \mathrm{U}=0.33$ $\varepsilon \mathrm{Hf}(\mathrm{t})$ $+0.61$
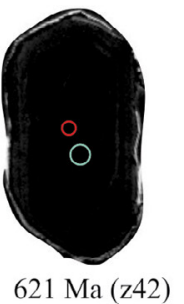

$\mathrm{Th} / \mathrm{U}=0.56$

$\varepsilon H f(t)$

$-2.10$

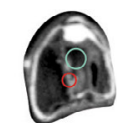

$783 \mathrm{Ma} \mathrm{(z20)}$

$\mathrm{Th} / \mathrm{U}=1.33$

$\varepsilon \mathrm{Hf}(\mathrm{t})$

$+1.17$
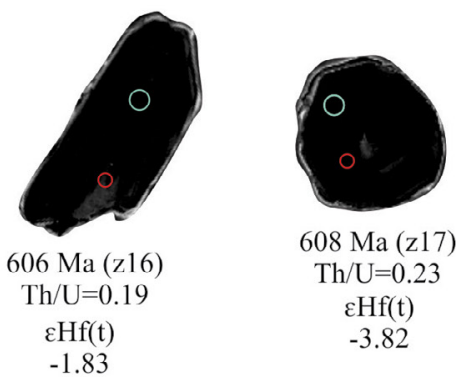

$\mathrm{Th} / \mathrm{U}=0.23$

$\varepsilon \mathrm{Hf}(\mathrm{t})$

$-3.82$

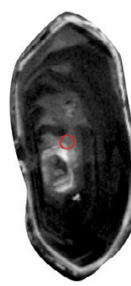

$648 \mathrm{Ma} \mathrm{(z04)}$

$\mathrm{Th} / \mathrm{U}=0.43$

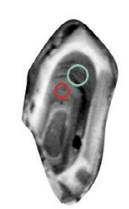

$614 \mathrm{Ma} \mathrm{(z19)}$

$\mathrm{Th} / \mathrm{U}=0.58$

$\varepsilon \mathrm{Hf}(\mathrm{t})$

$-0.06$

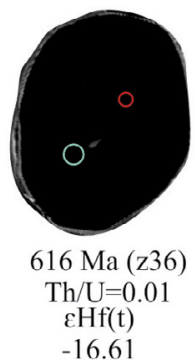

$624 \mathrm{Ma}(\mathrm{z} 10)$

$\mathrm{Th} / \mathrm{U}=0.43$

$\varepsilon \mathrm{Hf}(\mathrm{t})$

$\varepsilon H f(t)$
+3.53

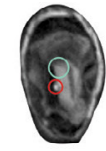

$789 \mathrm{Ma}(\mathrm{z} 03)$

$\mathrm{Th} / \mathrm{U}=0.24$

$\varepsilon \mathrm{Hf}(\mathrm{t})$

$\begin{array}{r}+0.69 \\ \hline\end{array}$
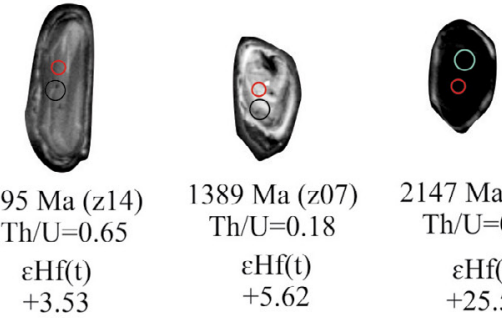

$1389 \mathrm{Ma}(\mathrm{z} 07)$

$\mathrm{Th} / \mathrm{U}=0.18$

$\varepsilon \mathrm{Hf}(\mathrm{t})$

$+5.62$

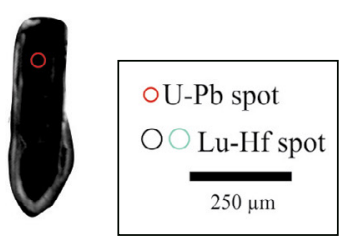

$674 \mathrm{Ma}(\mathrm{z} 28)$

$\mathrm{Th} / \mathrm{U}=0.29$

Figure 10. Hf evolution diagram for the analysed zircons. The blue ellipse highlights data for zircons defining the crystallization age, while the red ellipse shows the data for metamorphic zircons. A single datum for the Mesoproterozoic inherited zircon grain is presented in yellow. Selected images with both the $\mathrm{U}-\mathrm{Pb}$ and $\mathrm{Lu}-\mathrm{Hf}$ targets and $\mathrm{Th}-\mathrm{U}$ ratios are presented below. As expected for high-grade metamorphism the analysed zircons do not have low Th/U ratios. 
After evaporation Sm, Nd and Sr samples were separately loaded onto previously degassed Re filaments assembled in double filament mounts, using $\mathrm{H} 3 \mathrm{PO} 4$ as ionization activator.

Isotope ratios were measured with a Thermo Scientific TRITON in static mode using up to 8 Faraday collectors. The measured isotope ratios are reported with absolute standard errors $(2 \sigma)$ below 0.00001 . The measured ratios were normalized respectively to the natural constant ratios of ${ }^{146} \mathrm{Nd} /{ }^{144} \mathrm{Nd}=0.7219,{ }^{147} \mathrm{Sm} /{ }^{152} \mathrm{Sm}=0.5608$ and ${ }^{88} \mathrm{Sr} /{ }^{86} \mathrm{Sr}$ $=8.3762$. The average ${ }^{143} \mathrm{Nd} /{ }^{144} \mathrm{Nd}$ ratio of repeated measurements of the JNdi-1 reference material (Tanaka et al.2000) is $0.512098 \pm 0.000006(\mathrm{n}=322)$. The average ${ }^{87} \mathrm{Sr} /{ }^{86} \mathrm{Sr}$ ratio of the NBS-987 reference material (Wise \& Waters 2007) is $0.710239 \pm 0.000007(n=158)$. Repeated analyses of the BCR and AVG rock reference materials from the United States Geologic Survey yield ${ }^{147} \mathrm{Sm} /{ }^{144} \mathrm{Nd}$ ratios with reproducibility within 1\% (Valeriano et al. 2008). Neodymium $\left(\mathrm{T}_{\mathrm{DM}}\right)$ model ages were calculated using the depleted mantle model of De Paolo (1981).

\section{$\mathrm{Sm}-\mathrm{Nd}$ and $\mathrm{Sr}$ isotopes results}

The location of samples is shown in Figures 3 and 4, and the analytical results are presented in Table 4. Two isotopic groups can be discriminated. The majority of samples, including all those from the Barreiro suite, part of those from the Juiz de Fora Complex, and one single sample occurring within the metasedimentary rocks of Andrelândia-Raposos group yielded late Paleoproterozoic to Mesoproterozoic $\mathrm{T}_{\mathrm{DM}}$ model ages, between ca. $1.80 \mathrm{Ga}$ and $1.50 \mathrm{Ga}$. The $\varepsilon \mathrm{Nd}_{\mathrm{t}}$ values obtained are -8 to -3 , and initial ${ }^{87} \mathrm{Sr} /{ }^{86} \mathrm{Sr}$ ratios between 0.7017 and 0.7092 . This range of values suggests different degrees of contamination from country rocks, as indicated by inherited zircons with old U-Pb ages.

Sample RE-CM-28C, with highest Mg number, shows less contaminated isotopic signatures, probably reflecting the primary composition of the mafic magma with low $\mathrm{SiO}_{2}$ and high $\mathrm{MgO}$ contents. The young $\mathrm{T}_{\mathrm{DM}}$ age of $0.97 \mathrm{Ga}, \varepsilon_{\mathrm{t}}=$ +4.1 and initial ${ }^{87} \mathrm{Sr} /{ }^{86} \mathrm{Sr} 0.7036$ of this sample characterize it as the most primitive sample of the Barreiro Suite (Fig. 11).
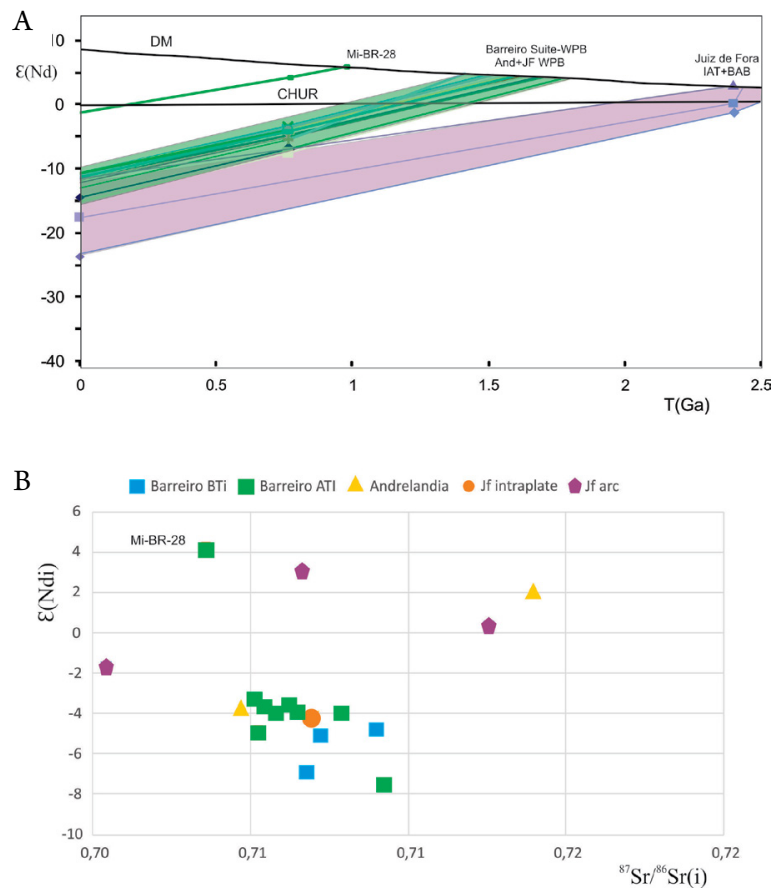

Figure 11. Sm-Nd isotopic data for selected analysed samples. (A) $\mathrm{Nd}$ evolution diagram; (B) $\varepsilon \mathrm{\varepsilon nd}_{(\mathrm{i})} \times{ }^{87} \mathrm{Sr} /{ }^{86} \mathrm{Sr}_{(\mathrm{i})}$ diagram for the samples of Barreiro Suite and a few mafic enclaves from the Andrelândia Group and Juiz de Fora Complex.

Table 4. Sm-Nd and $\mathrm{Sr}$ isotopic data of the metabasic rocks

\begin{tabular}{|c|c|c|c|c|c|c|c|c|c|c|c|c|c|c|c|c|c|}
\hline Sample & Unit & $\begin{array}{r}\text { Sm } \\
\text { ppm }\end{array}$ & $\begin{array}{c}\text { Nd } \\
\text { ppm }\end{array}$ & $\begin{array}{l}\mathbf{R b}^{*} \\
\mathbf{p p m}\end{array}$ & $\begin{array}{c}\text { Sr } \\
\text { ppm }\end{array}$ & $\begin{array}{l}{ }^{143} \mathrm{Nd} / \\
{ }^{144} \mathrm{Nd}\end{array}$ & $\begin{array}{c}\text { abs } \\
\text { st error }\end{array}$ & $\begin{array}{l}{ }^{147} \mathrm{Sm} / \\
{ }^{144} \mathrm{Nd}\end{array}$ & $\begin{array}{l}{ }^{87} \mathrm{Sr} / \\
{ }^{86} \mathrm{Sr}\end{array}$ & $\begin{array}{c}\text { abs } \\
\text { st error }\end{array}$ & $\begin{array}{c}\mathbf{t} \\
(\mathrm{Ma}) \\
\end{array}$ & $\begin{array}{c}\varepsilon \\
\mathbf{0 M a} \\
\end{array}$ & $\begin{array}{c}\mathbf{f} \\
(\mathrm{Sm} / \mathbf{N d})\end{array}$ & $\begin{array}{l}{ }^{143} \mathbf{N d} / \\
{ }^{144} \mathbf{N d}_{(t)}\end{array}$ & $\begin{array}{l}\varepsilon \\
(t)\end{array}$ & $\begin{array}{l}\mathbf{T}_{\mathrm{DM}} \\
\mathbf{G a}\end{array}$ & $\begin{array}{l}{ }^{87} \mathbf{S r} / \\
{ }^{86} \mathbf{S r} /(t)\end{array}$ \\
\hline RE-CE-03A & Barreiro LTi & 6.2 & 31.1 & 9 & 626 & 0.511899 & 0.000005 & 0.1200 & 0.707183 & 0.000006 & 766 & -14.4 & -0.38972 & 0.511296 & -6.9 & 1.76 & 0.7067 \\
\hline RE-CE-149 & Barreiro LTi & 4.6 & 21.5 & 6 & 641 & 0.512056 & 0.000008 & 0.1296 & 0.709232 & 0.000008 & 766 & -11.4 & -0.34095 & 0.511405 & -4.8 & 1.69 & 0.7089 \\
\hline RE-BR-68 & Barreiro LTi & 8.1 & 39.2 & 5 & 427 & 0.512018 & 0.000007 & 0.1257 & 0.707539 & 0.000004 & 766 & -12.1 & -0.36087 & 0.511387 & -5.1 & 1.68 & 0.7072 \\
\hline RE-CE-2A & Barreiro HTi & 9.9 & 46.6 & 8 & 507 & 0.512094 & 0.000005 & 0.1286 & 0.706950 & 0.000009 & 766 & -10.6 & -0.34611 & 0.511448 & -4.0 & 1.61 & 0.7065 \\
\hline RE-CE-13B & Barreiro HTi & 10.4 & 48.7 & 15 & 573 & 0.512049 & 0.000004 & 0.1288 & 0.706041 & 0.000005 & 766 & -11.5 & -0.34529 & 0.511402 & -4.9 & 1.68 & 0.7052 \\
\hline RE-CE-28C & Barreiro HTi & 5.7 & 24.4 & 15 & 556 & 0.512569 & 0.000004 & 0.1406 & 0.704435 & 0.000010 & 766 & -1.4 & -0.28519 & 0.511862 & 4.1 & 0.97 & 0.7036 \\
\hline RE-CE-29 & Barreiro HTi & 9.3 & 46.7 & 29 & 622 & 0.512088 & 0.000006 & 0.1209 & 0.706583 & 0.000007 & 766 & -10.7 & -0.38529 & 0.511481 & -3.3 & 1.50 & 0.7051 \\
\hline RE-CE-30A & Barreiro HTi & 8.3 & 41.7 & 13 & 553 & 0.512048 & 0.000006 & 0.1205 & 0.708587 & 0.000007 & 766 & -11.5 & -0.38746 & 0.511443 & -4.1 & 1.55 & 0.7078 \\
\hline RE-CE-30B & Barreiro HTi & 10.5 & 53.5 & 7 & 569 & 0.511861 & 0.000005 & 0.1188 & 0.709596 & 0.000004 & 766 & -15.2 & -0.39591 & 0.511264 & -7.6 & 1.80 & 0.7092 \\
\hline RE-CE-70B & Barreiro HTi & 10.0 & 47.9 & 6 & 619 & 0.512091 & 0.000003 & 0.1267 & 0.705819 & 0.000009 & 766 & -10.7 & -0.35601 & 0.511454 & -3.8 & 1.58 & 0.7055 \\
\hline RE-CE-27C & Barreiro HTi & 14.8 & 74.3 & 24 & 498 & 0.512070 & 0.000006 & 0.1204 & 0.707729 & 0.000006 & 766 & -11.1 & -0.38794 & 0.511465 & -3.6 & 1.52 & 0.7062 \\
\hline RE-CE-13B & Barreiro HTi & 10.3 & 48.6 & 15 & 573 & 0.512040 & 0.000009 & 0.1288 & 0.706054 & 0.000009 & 766 & -11.7 & -0.34542 & 0.511394 & -5.0 & 1.70 & 0.7052 \\
\hline RE-CE-19 & Barreiro HTi & 8.5 & 39.7 & 3 & 657 & 0.512091 & 0.000005 & 0.1291 & 0.705865 & 0.000006 & 766 & -10.7 & -0.34367 & 0.511443 & -4.1 & 1.62 & 0.7057 \\
\hline MI-BE-87B & Andrelândia & 8.2 & 48.6 & 57 & 711 & 0.511970 & 0.000006 & 0.1204 & 0.707192 & 0.000008 & 766 & -13.0 & -0.38794 & 0.511365 & -3.7 & 1.41 & 0.7047 \\
\hline MI-BR-39 & Andrelândia & 3.0 & 9.6 & 7 & 146 & 0.512692 & 0.000007 & 0.1870 & 0.715332 & 0.000037 & 766 & 1.1 & -0.04929 & 0.511753 & 2.0 & 1.69 & 0.7138 \\
\hline MI-III-18 & $\begin{array}{c}\text { Juiz de Fora } \\
\text { WPB }\end{array}$ & 10.0 & 48.4 & 9 & 639 & 0.512059 & 0.000008 & 0.1245 & 0.707242 & 0.000006 & 766 & -11.3 & -0.36717 & 0.511433 & -4.2 & 1.60 & 0.7068 \\
\hline $\begin{array}{l}\text { PA-BE- } \\
137 A\end{array}$ & $\begin{array}{c}\text { Juiz de Fora } \\
\text { IAT }\end{array}$ & 8.0 & 38.8 & 72 & 464 & 0.511425 & 0.000009 & 0.1254 & 0.715975 & 0.000002 & 2400 & -23.7 & -0.36247 & 0.509441 & -1.7 & 2.58 & 0.7004 \\
\hline MI-BR-19C & $\begin{array}{c}\text { Juiz de Fora } \\
\text { IAT }\end{array}$ & 6.1 & 26.5 & 12 & 250 & 0.511734 & 0.000005 & 0.1386 & 0.717248 & 0.000008 & 2400 & -17.6 & -0.29543 & 0.509542 & 0.3 & 2.43 & 0.7124 \\
\hline MI-VIII-15 & $\begin{array}{c}\text { Juiz de Fora } \\
\text { IAT }\end{array}$ & 4.2 & 17.2 & 2 & 35 & 0.512047 & 0.000008 & 0.1496 & 0.712324 & 0.000009 & 2400 & -11.5 & -0.23962 & 0.509681 & 3.0 & 2.16 & 0.7066 \\
\hline
\end{tabular}

$\mathrm{Rb}^{*}$-Values from geochemical data 
Three mafic enclaves with geochemical signatures of convergent tectonic settings (IAT/BAB or CAB), found in the Juiz de Fora Complex, yielded $\mathrm{T}_{\mathrm{DM}}$ ages between $2.58 \mathrm{Ga}$ and $2.16 \mathrm{Ga}$, with $\varepsilon_{\mathrm{t}}$ between +3.0 and -1.7 . These samples display initial ${ }^{87} \mathrm{Sr} /{ }^{86} \mathrm{Sr}$ ratios between 0.7004 and 0.7124 . These results reflect an older convergent juvenile accretion history of the Juiz de Fora complex, as previously reported by Heilbron et al. (2010) and Degler et al. (2018).

\section{DISCUSSION}

\section{Two tectonic settings in the studied area indicated by metabasic rocks}

The data presented above, summarized in Table 5, suggest late Tonian (ca. $766 \mathrm{Ma}$ ) extensional intraplate magmatism both in the cover and in the basement of the distal segment of the São Francisco paleocontinent passive margin (Andrelândia Group).

The development of the Andrelândia basin in the proximal zone started in the Mesoproterozoic to Neoproterozoic transition, as indicated by the youngest detrital zircons of ca. $0.9 \mathrm{Ma}$ (Valeriano et al.2004, Belém et al. 2011) and $\mathrm{T}_{\mathrm{DM}}$ of interlayered metabasic rocks of ca. $1.0 \mathrm{Ga}$ (Heilbron et al. 1989, Frugis \& Campos Neto 2018). The new data presented here indicate that the evolution of the distal passive margin lasted at least until late Tonian times.

This late Tonian within-plate magmatism displays within plate basalts (WPB) signatures, with both high- $\mathrm{TiO}_{2}$ and low$\mathrm{TiO}_{2}$ groups, with a few samples showing relatively primitive MORB-like signatures. Mafic magmatic rocks intrude both basement rock associations (Juiz de Fora Complex), as well the cover of distal Andrelândia passive margin (Figs. 12 and 13).

A few samples detected only in the Juiz de Fora Complex display convergent tectonic signatures (IAT to CAB) and older

Table 5. Summary of the new data obtained for mafic rocks of the Barreiro Suite and mafic enclaves of Andrelândia Group and Juiz de Fora Complex.

\begin{tabular}{|c|c|c|c|c|c|c|}
\hline Mafic groups & & U-Pb age $(\mathbf{G a})$ & $\mathbf{T}_{\mathrm{DM}(\mathrm{Nd})}(\mathbf{G a})$ & $\varepsilon N d_{t}$ & ${ }^{87} \mathbf{S r} /{ }^{86} \mathbf{S r}(\mathbf{i})$ & $\varepsilon \mathrm{Hf}_{\mathrm{t}}$ and $\mathrm{T}_{\mathrm{DM}(\mathrm{Hf})}$ \\
\hline $\begin{array}{l}\text { High } \mathrm{TiO}_{2} \text { Barreiro Suite and } \\
\text { few mafic enclaves }\end{array}$ & Intraplate & & 1.80 to 1.50 & -8 to -3 & 0.7017 to 0.7092 & \\
\hline $\begin{array}{l}\text { Low } \mathrm{TiO}_{2} \text { Barreiro suite and } \\
\text { enclaves of Juiz de Fora Complex } \\
\text { and Andrelândia Group }\end{array}$ & Intraplate & 0.77 & 1.80 to 1.50 & -8 to -3 & 0.7017 to 0.7092 & $\begin{array}{l}1.75 \text { to } 0.96 \mathrm{Ga}, \\
+5.61 \text { to }+0.24\end{array}$ \\
\hline $\begin{array}{l}\text { Most primitive sample of the } \\
\text { Barreiro Suite (RE-BR-28) }\end{array}$ & Intraplate & & 0.97 & +4 & 0.7036 & \\
\hline $\begin{array}{l}\text { Low } \mathrm{TiO}_{2} \text { enclaves of the } \\
\text { Andrelândia group and Juiz de } \\
\text { Fora Complex }\end{array}$ & Morb & & 1.80 to 1.50 & -8 to -3 & 0.7017 to 0.7092 & \\
\hline $\begin{array}{l}\text { Low } \mathrm{TiO}_{2} \text { enclaves of Juiz de } \\
\text { For a complex }\end{array}$ & $\begin{array}{l}\text { IATand } \\
\text { CAB }\end{array}$ & 2.2 to 2.07 & 2.58 to 2.16 & +3 to -1.7 & 0.7004 to 0.7124 & \\
\hline
\end{tabular}

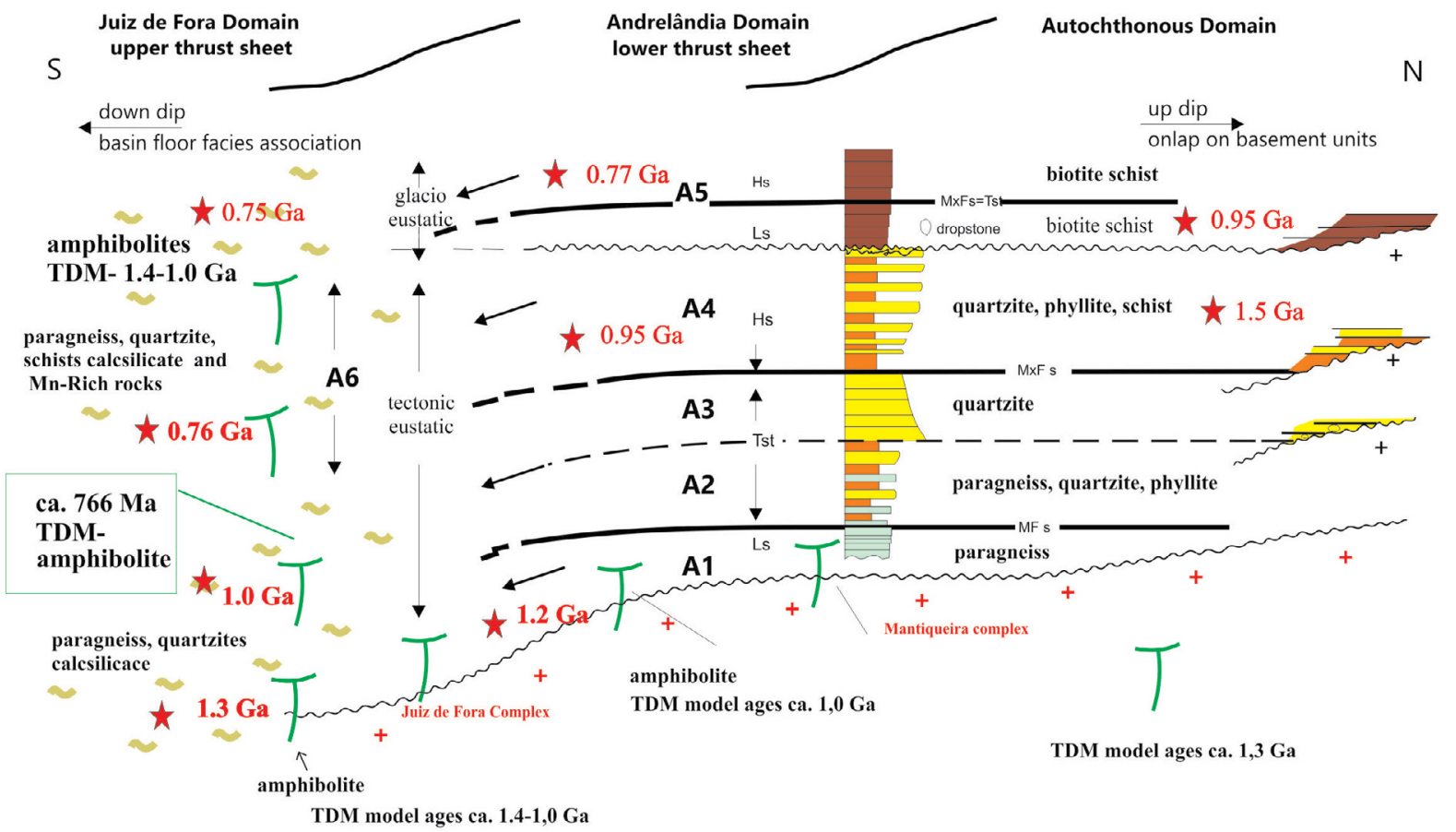

Figure 12. Composite stratigraphic chart for the Andrelândia basin, including proximal (autochthonous and Andrelândia domains) and distal (Andrelândia and Juiz de Fora domains), modified from Ribeiro et al. (2013) and Heilbron et al. (2017a). Amphibolite data are from this work, Frugis \& Campos Neto (2018) and Heilbron et al. (1989). Younger detrital zircon was compiled from Valladares et al. (2004, 2008), Ribeiro et al. (2013), Degler et al. (2017), Frugis \& Campos Neto (2018). 
$\mathrm{T}_{\mathrm{DM}}$ model ages, indicating that they probably belong to the Paleoproterozoic history of the basement.

Both associations show evidence of Neoproterozoic Brasiliano overprinting, with high deformation textures and granulite facies metamorphism between ca. 619 (this work) and $565 \mathrm{Ma}$ (Duarte 2003, Heilbron \& Machado 2003, Bento dos Santos et al. 2007, Degler et al. 2017).

\section{Correlation with previous data reported for the Andrelândia basin}

Previous works have reported combined geochemical and geochronological data for the metabasic rocks interlayered with the metasedimentary rocks of the Andrelândia Basin. In the most proximal region, between Madre de Deus de Minas and Andrelândia towns, southern Minas Gerais State, Gonçalves \& Figueiredo (1992) and Paciullo et al. (2000) obtained very similar results, with low- and high- $\mathrm{TiO}_{2}$ amphibolites, pointing to intraplate to MORB-like signatures, suggestive of syn-sedimentary magmatism. These authors suggested that the magmatism evolved in time from high- $\mathrm{TiO}_{2}$ intraplate rocks in the lower units to MORB signatures in the upper units of the basin. Marins (2000) also reported the same geochemical groups for the amphibolites interlayered within the distal portion of the Andrelândia basin (Raposos Group). The comparison between all the available geochemical data is presented in Figure 13 and provides additional evidence of a major episode of intraplate to E-MORB tholeiitic magmatism between $0.9 \mathrm{Ga}$ and 0.77
$\mathrm{Ga}$ associated with the development of the southeastern and eastern São Francisco paleocontinent passive margin.

\section{Tectonic implications for the Neoproterozoic passive margin evolution within the Araçuaí-Ribeira orogenic system}

The geochronology data presented herein provide constraints to the timing of evolution of the distal segment of the Andrelândia basin (Raposos Group) from ca. 1.0 Ga to ca. $766 \mathrm{Ma}$ (Fig. 12). This age interval agrees well with the evolution of the Macaúbas Group, located to the north, in the Araçuaí Belt. Other reported coeval intraplate magmatic episodes are the Pedro Lessa suite, the Northern Espinhaço mafic dykes and the Salto da Divisa A-type granites, with ages of 910 to $850 \mathrm{Ma}$, as well as correlative bimodal volcanic rocks and A-type granites in Africa with ages of 930 to $910 \mathrm{Ma}$ (PedrosaSoares \& Alkmim 2011, Pedrosa-Soares et al. 2000, 2016).

Published ages between ca. $735 \mathrm{Ma}$ and $675 \mathrm{Ma}$, from the Southern Bahia Alkaline Province, and from the La Louila felsic volcanism in southwest Gabon ( $\leq 713 \mathrm{Ma})$ constrain the timing of deposition of the top units of the Macaúbas Group and African correlatives (Pedrosa-Soares \& Alkmim 2011). This data indicates that the extensional history of the São Francisco eastern passive margin could have lasted until Cryogenian times, coeval with the development of outboard magmatic arcs eastern wards (Heilbron et al. 2008, 2017a).

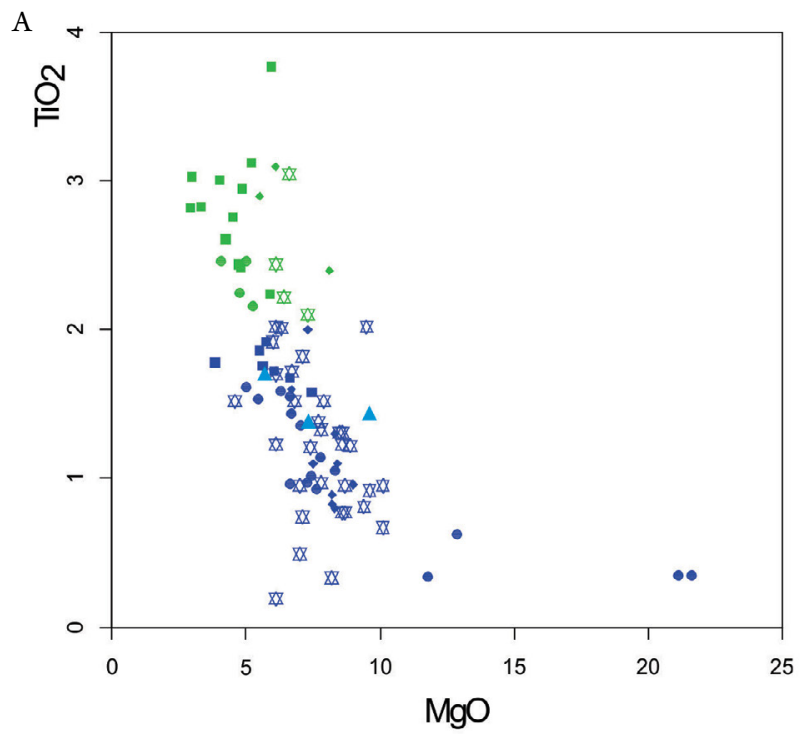

B

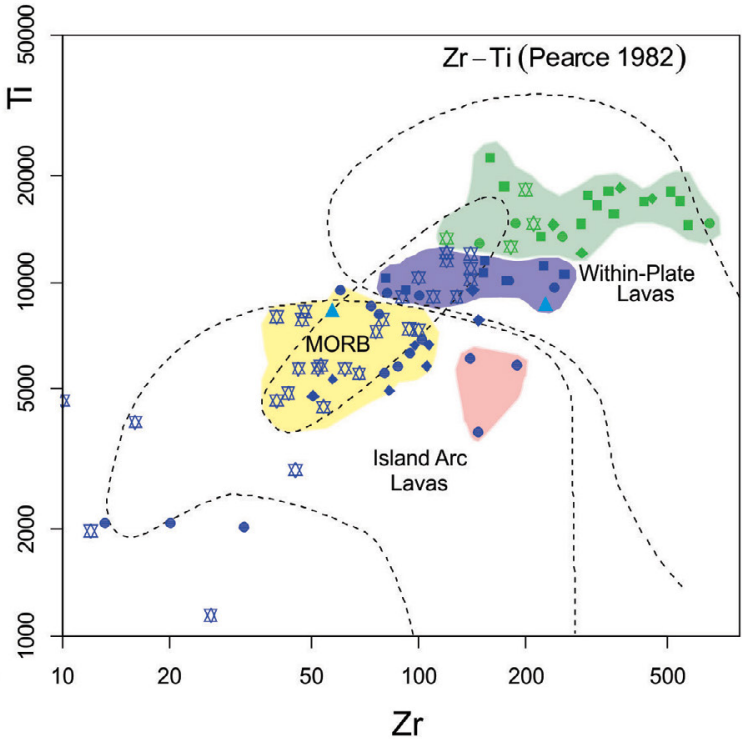

\begin{tabular}{|c|c|c|}
\hline Symbols & \multicolumn{2}{|c|}{ Low- $\mathrm{TiO}_{2}$ High- $\mathrm{TiO}_{2}$} \\
\hline Barreiro Suite & ! & ! \\
\hline Juiz de Fora enclaves & $\Delta$ & $\Delta$ \\
\hline Distal Andrelândia Group enclaves & $\Delta$ & \\
\hline $\begin{array}{l}\text { Proximal Andrelândia Group enclaves } \\
\text { stars-Paciullo et al, 1997; losangles-Gonçalves e Figueiredo, } 1992\end{array}$ & ${ }_{92} \not 又<$ & $B D$ \\
\hline
\end{tabular}

Figure 13. A comparison of new data and previously published data for mafic rocks in the region of the Andrelândia deformed basin units. Proximal data are compiled from Paciullo (1997) and Gonçalves and Figueiredo (1992), compared with our data for the distal part of the basin (Barreiro Suite) and the mafic enclaves of both the Andrelândia Group and the Juiz de Fora Complex. (A) $\mathrm{TiO}_{2} \times \mathrm{MgO}_{\text {diagram }}$ showing that both proximal and distal samples could be subdivided into the same groups, and (B) plot in the Zi-Ti tectonic diagram of Pearce (1982), with green, blue, yellow and pink fields to show respectively, the intraplate High- and $\mathrm{Low}_{-} \mathrm{TiO}_{2}$ groups, MORB-like samples and a few older mafic enclaves of the Juiz de Fora Complex with arc-related signatures. 


\section{CONCLUSIONS}

The combination of the data presented herein with previously reported studies for the metabasic rocks of the Ribeira Belts reveals that at least three different episodes of mafic magmatism can be detected in the distal part of the Andrelândia basin and associated basement complexes. The two older episodes are represented by dismembered metabasic rocks found within the orthogranulites of the basement Juiz de Fora Complex and related to the Rhyacian arc (ca.2.2 to 2.1 Ga) and to Statherian (ca. $1.7 \mathrm{Ga}$ ) intraplate magmatism. The youngest episode occurs as dismembered metabasic bodies that intruded both the cover and the basement association, with a late Tonian age of ca. $766 \mathrm{Ma}$, and clearly displays intraplate signatures associated with the evolution of the distal passive margin (Fig. 13).

An important implication of the characterization of this Late Tonian to Early Cryogenian intraplate metabasic rocks is that they must have shed detrital zircons that have been found sparsely in previous studies on the distal segments of the Andrelândia basin. We suggest that the existence of detrital zircons originated from intraplate magmatic events should be considered in future provenance studies of metasedimentary units of Neoproterozoic succession around the São Francisco craton, rather than necessarily originating from approaching magmatic arcs, as they have been commonly interpreted.

\section{ACKNOWLEDGEMENTS}

The authors are grateful to CPRM-PRONAGEO, CNPq and FAPERJ for research funding, and to the technicians of the laboratories from Brasília (UnB-Brazil), LAGIR (UERJBrazil) and Notre Dame (USA) universities. Monica Heilbron and Marcela Lobato were granted respectively Senior Visiting Professor and Sandwich PhD CAPES scholarships. Claudio Valeriano acknowledges a scholarship from CNPq (Estágio Senior no Exterior). We also would like to thank the generous revisions of Brendan Murphy, Robert Pankhurst and a third anonymous reviewer, which helped to improve an earlier version of the manuscript.

\section{ARTICLE INFORMATION}

Manuscript ID: 20180129. Received on: 11/20/2018. Approved on: 02/19/2019.

M. H. wrote the manuscript, managed the corrections, made the final format of figures and maps, organized and integrated all data, collected samples and did the fieldwork. C. O. contributed to write the manuscript, obtained and discussed the geochemical data, including figures and tables. M. L. discussed the data, ran the U-Pb and Lu-Hf data, made figures and respective tables. I. D. contributed to write the manuscript, helped to process and discussed U-Pb and Lu-Hf. C. V. contributed to write and revise the manuscript, obtained and discussed Sm-Nd data, including tables and figures. E. D. obtained and helped to process and discuss the U-Pb data. A. S. obtained and helped to process and discuss the Hf data. H. B. contributed to field work, maps and geochemical approach, including final version of the figures of geochemistry. F. C. contributed to field work, maps and geochemical approach collected and processed samples and geochronology approach. E. S. contributed to field work and made the geology maps and structural geology approach.

Competing interests: The authors declare no competing interests.

\section{REFERENCES}

Alkmim F.F, Kuchenbecker M., Reis H.L.S., Pedrosa-Soares A.C. 2017. The Araçuaí belt. In: Heilbron M., Cordani U.G., Alkmim F.F. (eds). São Francisco Craton, Eastern Brazil Tectonic Genealogy of a Miniature Continent. 1 ed. Springer, v. 1, 251-276.

Almeida F.F.M. de, Hasui Y., Brito Neves B.B., Fuck R.A. 1981. Brazilian structural provinces: an introduction. Earth-Science Reviews, 17(1-2):1-29. https://doi.org/10.1016/0012-8252(81)90003-9

Andersen T., Andersson U.B., Graham S., Åberg G., Simonsen S.L. 2009. Granitic magmatism by melting of juvenile continental crust: new constraints on the source of Paleoproterozoic granitoids in Fennoscandia from Hf isotopes in zircon. Journal of the Geological Society, 166(2):233-247. http://dx.doi.org/10.1144/0016-76492007-166

Belém J., Pedrosa-Soares A.C., Noce C.M., Silva L.C., Armstrong R., Fleck A., Gradim C.T., Queiroga G.N. 2011. Bacia precursora versus bacias orogênicas: exemplos do Grupo Andrelândia com base em datações U-Pb (LA-ICP-MS) em zircão e análises litoquímicas. Geonomos, 19(2):224-243. https://doi.org/10.18285/geonomos.v19i2.55

Bento dos Santos T., Munhá J., Tassinari C., Fonseca P., Dias Neto C. 2007. Thermochronological evidence for long-term elevated geothermal gradients in Ribeira Belt, SE Brazil. Geochimica et Cosmochimica Acta, 71(15 Suppl. 1):A79.

Bouvier A., Vervoort J.D., Patchett P.J. 2008. The Lu-Hf and Sm-Nd isotopic composition of CHUR: Constraints from unequilibrated chondrites and implications for the bulk composition of terrestrial planets. Earth and
Planetary Science Letters, 273(1-2):48-57. https://doi.org/10.1016/j. eps1.2008.06.010

Boynton WV. 1984. Geochemistry of Rare Earth Elements: Meteorite Studies. In: Henderson P. (eds). Rare Earth Element Geochemistry. Elsevier, New York, p. 63-114.

Bühn B., Pimentel M.M., Matteini M., Dantas E. 2009. High spatial resolution analysis of $\mathrm{Pb}$ and $\mathrm{U}$ isotopes for geochronology by laser ablation multicollector inductively coupled plasma mass spectrometry inductively coupled plasma mass spectrometry (LA-MC-ICPMS). Anais da Academia Brasileira de Ciências, 81 (1):99-114. http://dx.doi.org/10.1590/ S0001-37652009000100011

Campos Neto M.C. 2000. Orogenic systems from Southwestern Gondwana, an approach to Brasiliano-Pan African cycle and orogenic collage in southeastern Brazil. In: Cordani U.G., Milani E.J., Thomaz Filho A., Campos D.A. (eds.), Tectonic Evolution of South America. Rio de Janeiro, SBG.

Campos Neto M.C., Basei M.A.S., Janasi V.A., Moraes R. 2011. Orogen migration and tectonic setting of the Andrelândia Nappe system: an Ediacaran western Gondwana collage, south of São Francisco Craton. Journal of South American Earth Sciences. 32(4):393-406.

Campos Neto M.C., Basei M.A.S., Vlach S.R.F., Caby R., Szabo G.A.J., Vasconcelos P. 2004. Migração de orógenos e superposição de orogêneses: Um esboço da colagem brasiliana no sul do Cráton do São Francisco, SEBrasil. Boletim do Instituto de Geociências/USP, 4:13-40. 
Coelho M.B., Trouw R.A.J., Ganade de Araújo C.E., Vinagre R., Mendes J.C., Sato K. 2017. Constraining timing and P-T conditions of continental collision and late overprinting in the Southern Brasília Orogen (SE-Brazil): $\mathrm{U}-\mathrm{Pb}$ zircon ages and geothermobarometry of the Andrelândia Nappe System. Precambrian Research, 292:194-215. https://doi.org/10.1016/j. precamres.2017.02.001

Cox K.G. 1989. The role of mantle plumes in the development of continental drainage patterns. Nature, 342:873-877. http://dx.doi. org $/ 10.1038 / 342873 \mathrm{a} 0$

De la Roche H., Leterrier J., Grand Claude P., Marchal M. 1980. A classification of volcanic and plutonic rocks using R1--R2 diagrams and major element analyses - its relationships with current nomenclature. Chemical Geology., 29:183-210.

De Paolo D.J. 1981. A neodymium and strontium isotopic study of the Mesozoic calc-alkaline granitic batholiths of the Sierra Nevada and Peninsular Ranges, California. Journal of Geophysical Research, 86(B11):10470-10488. https://doi.org/10.1029/JB086iB11p10470

Degler R., Pedrosa-Soares A.C., Dussin I., Queiroga G., Schulz B. 2017. Contrasting provenance and timing of metamorphism from paragneisses of the Araçuaí-Ribeira orogenic system, Brazil: Hints for Western Gondwana assembly. Gondwana Research, 57:30-50. http://dx.doi.org/10.1016/j. gr.2017.07.004

Degler R., Pedrosa-Soares A.C., Novo T., Tedeschi M., Silva L.C., Dussin I., Lana C. 2018. Rhyacian-Orosirian isotopic records from the basement of theAraçuaí-Ribeira orogenic system (SE Brazil): Links in the CongoSão Francisco paleocontinent. Precambrian Research, 317:179-195. http:// dx.doi.org/10.1016/j.precamres.2018.08.018

Duarte B., Heilbron M., Nogueira J.R., Tupinambá M., Eirado L.G., Valladares C., Almeida J.C.H., Guia C. 2003. Geologia das Folhas Juiz de Fora e Chiador. In: Pedrosa-Soares A.C., Noce C.M., Trouw R., Heilbron M. (coords.). Projeto Sul de Minas. Belo Horizonte, COMIG/SEME, v. 1.p. $153-258$

Frugis G. \& Campos Neto M. 2018. Eastern Paranapanema and Southern São Francisco orogenic margins: Records of enduring Neoproterozoic oceanic convergence and collision in the Southern Brasília Orogen. Precambrian Research, 308:35-57.

Gonçalves M.L. \& Figueiredo M.C.H. 1992. Geoquímica dos Anfibolitos de Santana do Garambéu (MG): Implicações Tectônicas sobre a Evolução do Grupo Andrelândia. Geochimica Brasiliensis, 6(2):127-140.

Griffin W.L., Pearson N.J., Belousova E., Jackson S.E., van Achterbergh E., O'Reilly S.Y., Shee S.R. 2000. The Hf isotope composition of cratonic mantle: LAM-MC- ICPMS analysis of zircon megacrysts in kimberlites. Geochimica et Cosmochimica Acta, 64(1):133-147.https://doi.org/10.1016/ S0016-7037(99)00343-9

Heilbron H., Cordani U., Alkmim F., Reis H. 2017a. Tectonic Genealogy of a Miniature Continent. In: Heilbron M., Cordani U.G., Alkmim F.F. (eds.), São Francisco Craton, Eastern Brazil. Regional Geology Reviews. Berlin, Springer.

Heilbron M., Duarte B.P., Nogueira J.R. 1998. The Juiz de Fora complex of the Central Ribeira belt, SE Brazil: a segment of Paleoproterozoic granulitic crust thrust during the Pan-African Orogen. Gondwana Research, 1(3):373381. http://dx.doi.org/10.1016/S1342-937X(05)70853-4

Heilbron M., Duarte B.P., Valeriano C., Simonetti A., Machado N., Nogueira J. 2010. Evolution of reworked Paleoproterozoic basement rocks within the Ribeira belt (Neoproterozoic), SE-Brazil, based on $\mathrm{U} \mathrm{Pb}$ geochronology: Implications for paleogeography reconstructions of the São FranciscoCongo paleocontinent. Precambrian Research, 178(1):136-148. http:// dx.doi.org/10.1016/j.precamres.2010.02.002

Heilbron M., Gonçalves M.L., Teixeira W., Trouw R.A.J., Padilha A., Kawashita K. 1989. Geocronologia da área entre Lavras, São João del Rei, Lima Duarte e Caxambu. Anais da Academia Brasileira de Ciências, 61(2):177-199.

Heilbron M. \& Machado N. 2003. Timing of terrane accretion in the Neoproterozoic-Eopaleozoic Ribeira belt (se Brazil). Precambrian Research, 125(1-2):87-112.

Heilbron M., Pedrosa-Soares A.C., Campos Neto M., Silva L.C., Trouw R.A.J., Janasi V.C. 2004. A Província Mantiqueira. In: Mantesso-Neto V., Bartorelli A., Carneiro C.D.R., Brito Neves B.B. (eds.), O Desvendar de um
Continente: A Moderna Geologia da América do Sul e o Legado da Obra de Fernando Flávio Marques de Almeida. São Paulo, Beca, p. 203-204

Heilbron M., Ribeiro A., Valeriano C.M., Paciullo F., Almeida J.C.H., Trouw R., Tupinambá M., Silva L.G.E. 2017a. The Ribeira belt. In: Heilbron M., Cordani U.G., Alkmim F. (eds.), São Francisco Craton, Eastern Brazil Tectonic Genealogy of a Miniature Continent. Berlin, Springer, v. 1, p. 277-304.

Heilbron M., Tupinambá M., Almeida J.C.H., Valeriano C., Gontijo A., Silva T.M., Menezes P.T.L., Mane M., Palermo N., Pereira R.M. 2012. Introdução. In: Heilbron M. (eds.), Geologia e recursos minerais da folha Santo Antônio de Pádua SF. 26-X-D-VI, escala 1:100.000. Belo Horizonte, CPRM, v. 1, p. 17-21.

Heilbron M., Valeriano C.M., Almeida J.C.H., Eirado L.G. 2107b. Geologia e Recursos Minerais do estado do Rio de Janeiro. 1. ed. Brasília, CPRM, v. 1, 182p.

Heilbron M., Valeriano C.M., Tassinari C.C.G., Almeida J., Tupinambá M., Siga O., Trouw R. 2008. Correlation of Neoproterozoic terranes between the Ribeira Belt, SE Brazil and its African counterpart: comparative tectonic evolution and open questions. Geological Society Special Publications, 294:211-237. https://doi.org/10.1144/SP294.12

International Union of Pure and Applied Chemistry. 1998. Commission on Atomic Weights and Isotopic Abundances. Isotopic Compositions of the Elements 1997. Pure and Applied Chemistry, 70(1):217-235.

Irvine T.N. \& Baragar W.R.A. 1971. A guide to the chemical classification of the common volcanic rocks. Canadian Journal of Earth Sciences, 8(5):523548. https://doi.org/10.1139/e71-055

Janoušek V., Farrow C.M., Erban V. 2006. Interpretation of whole-rock geochemical data in igneous geochemistry: introducing Geochemical Data Toolkit (GCDkit). Journal of Petrology, 47(6):1255-1259. https://doi. org/10.1093/petrology/egl013

Le Bas M.J., Le Maitre R.W., Streckeisen A., Zanettin B. 1986. A chemical classification of volcanic rocks based on the total alkali-silica diagram. Journal of Petrology, 27(3):745-750. http://dx.doi.org/10.1093/ petrology/27.3.745

Le Maitre R.W., Bateman P., Dudek A., Keller J., Lameyre J., LeBas M.J., Sabine P.A., Schmid R., Sorensen H., Streckeisen A., Wooley A.R., Zanettin B. 1989. A classification of igneous rocks and glossary of terms. Oxford, Blackwell.

Ludwig K.R. 2003. Isoplot 3.00, A Geochronological Toolkit for Excel: Berkeley Geochronology Center Special Publication No. 4.

Machado N., Valladares C., Heilbron M., Valeriano C. 1996. U/Pb Geochronology Of Central Ribeira Belt: Implications For The Evolution Of Brasiliano Orogeny. Precambrian Research, 79(3):347-361.

Marins G. 2000. Petrologia dos Anfibolitos do Domínio Juiz de Fora e da Klippe Paraíba do Sul, no Setor Central da Faixa Ribeira. MS Dissertation, Programa em Análise de Bacias e Faixas Móveis, Universidade do Estado do Rio de Janeiro, Rio de Janeiro.

Meschede M. 1986. A method of discriminating between different types of mid-ocean ridge basalts and continental tholeiites with the $\mathrm{Nb}-\mathrm{Zr}-\mathrm{Y}$ diagram. Chemical Geology, 56:207-218.

Noce C.M., Pedrosa-Soares A.C., Silva L.C., Armstrong R., Piuzana D. 2007. Evolution of polycyclic basement complexes in the Araçuaí orogen, based on U-Pb SHRIMP data: Implication of Brazil-Africa links in Paleoproterozoic time. Precambrian Research, 159:60-78.

Noce C.M., Romano A.W., Pinheiro C.M., Mol V.S., Pedrosa-Soares A.C. 2003. Geologia das Folhas Ubá e Muriaé. In: Pedrosa-Soares A.C. Noce CM, Trouw R, Heilbron M.. (eds.), Geologia e Recursos Minerais do Sudeste Mineiro. Belo Horizonte, COMIG, p. 623-659.

Paciullo F.V.P. 1997. A Sequência Deposicional Andrelândia. Doctoral Thesis, Instituto de Geociências, Universidade Federal do Rio de Janeiro, Rio de Janeiro.

Paciullo F.V.P., Ribeiro A., Andreis R.R., Trouw R.A.J. 2000. The Andrelândia Basin, a Neoproterozoicintra-plate continental margin,southern Brasília Belt. Revista Brasileira de Geociências, 30(1):200-202.

Pearce J.A. 1982. Trace element characteristics of lavas from destructive plate boundaries. In: Thorpe R.S. (ed.), Andesites: orogenie andesites and related rocks. Chichester, Wiley, p. 525-548. 
Pearce J.A. 1983. The role of sub-continental lithosphere in magma genesis at destructive plate margins. In: Hawkesworth C.J. \& Norry M.J. (eds.), Continental basalts and mantle xenoliths. Nantwich: Shiva, p. 230-249.

Pearce J.A. 1987. An expert system for the tectonic characterization of ancient volcanic rocks. Journal of Volcanology and Geothermal Research, 32(1-3):51-65. https://doi.org/10.1016/0377-0273(87)90036-9

Pearce J.A. \& Cann J.R. 1973. Tectonic setting of basic volcanic rocks determined using trace element analysis. Earth and Planetary Science Letters, 19(2):290-300. http://dx.doi.org/10.1016/0012-821X(73)90129-5

Pearce T.H., Gorman B.E., Birkett T.C. 1975. The TiO2-K2O-P2O5 diagram: a method of discriminating between oceanic and non-oceanic basalts. Earth and Planetary Science Letters, 24(3):419-426. https://doi. org/10.1016/0012-821X(75)90149-1

Pedrosa-Soares A.C. \& Alkmim F.F. 2011. How many rifting events preceded the development of the Araçuaí-West Congo orogen? Geonomos, 19(2). https://doi.org/10.18285/geonomos.v19i2.56

Pedrosa-Soares A.C., Alkmim F.F., Tack L., Noce C.M., Babinski M., Silva L.C., Martins-Neto M. 2008. Similarities and differences between the Brazilian and African counterparts of the Neoproterozoic AraçuaíWest Congo Orogen. In: Pankhurst J.R., Trouw R.A.J., Brito Neves B.B., De Wit M.J. (eds.).West Gondwana: Pre-Cenozoic Correlations across the South Atlantic Region. Geological Society, London, Special Publications, 294, p. 153-172.

Pedrosa-Soares A.C., Dussin I., Nseka P., Baudet D., Fernandez-Alonso M., Tack L. 2016. Tonian rifting events on the Congo-São Francisco paleocontinent: New evidence from $\mathrm{U}-\mathrm{Pb}$ and $\mathrm{Lu}-\mathrm{Hf}$ data from the Shinkakasa plutonic complex (Boma region, West Congo Belt, Democratic Republic of Congo). In: International Geologica Belgica Meeting. Mons, 5., Belgium. Abstract Book..., p. 44.

Pedrosa-Soares A.C., Vidal P., Leonardos O.H., Brito-Neves B.B. 1998. Neoprotero-zoic oceanic remnants in eastern Brazil: further evidence and refutation of an exclusively ensialic evolution for the Araçuaí-West Congo orogen. Geology, 26(6):519-522. https://doi. org/10.1130/0091-7613(1998)026\%3C0519:NORIEB\%3E2.3.CO;2

Pedrosa-Soares A.C., Wiedemann-Leonardos C.M. 2000. Evolution of the Araçuaí Belt and its connection to the Ribeira Belt, Eastern Brazil. In: Cordani U., Milani E., Thomaz-Filho A., Campos D.A. (eds.), Tectonic Evolution of South America. São Paulo, Sociedade Brasileira de Geologia, p. 265-285.

Ribeiro A., Teixeira W., Dussin I.A., Ávila C.A., Nascimento D. 2013. U-Pb LA-ICP-MS detrital zircon ages of the São João del Rei and Carandaí basins: New evidence of intermittent Proterozoic rifting in the São Francisco paleocontinent. Gondwana Research, 24(2):713-726. http://dx.doi. org/10.1016/j.gr.2012.12.016

Ribeiro A., Trouw R.A.J, Andreis R.R., Paciullo F.V.P., Valença J.G. 1995. Evolução das bacias proterozóicas e o termo-tectonismo brasiliano na margem sul do cráton do São Francisco. Revista Brasileira de Geociências, 25:235-248.

Rollinson H. 1993. Using geochemical data: evaluation, presentation, interpretation. United Kingdom, Pearson Education Limited, Longman Group.

Rubatto D. 2017. Zircon: The Metamorphic Mineral. In: Kohn M.J., Engi M., Lanari P. (eds.), Petrochronology. Reviews in Mineralogy and Geochemistry, 83:261-289.

Saccani E. 2015. A new method of discriminating different types of postArchean ophiolitic basalts and their tectonic significance using Th-Nb and Ce-Dy-Yb systematics. Geoscience Frontiers, 6(4):481-501. https://doi. org/10.1016/j.gsf.2014.03.006

Shervais J.V. 1982. Ti-V plots and the petrogenesis of modern and ophiolitic lavas. Earth and Planetary Science Letters, 59:101-118.

Silva L.C., Armstrong R., Noce C.M., Carneiro M., Pimentel M., PedrosaSoares A.C., Leite C., Vieira V.S., Silva M., Paes V., Cardoso-Filho J. 2002. Reavaliação da evolução geológica em terrenos pré-cambrianos brasileiros com base em novos dados U-Pb SHRIMP; parte II: Orógeno Araçuaí, Cinturão Móvel Mineiro e Cráton São Francisco Meridional. Revista Brasileira de Geociências, 32(4):513-528.

Simonetti A., Neal C.R. 2010. In-situ chemical, U-Pb dating, and Hf isotope investigation of megacrystic zircons, Malaita (Solomon Islands): Evidence for multi-stage alkaline magmatic activity beneath the Ontong Java Plateau. Earth and Planetary Science Letters, 295:251-261. http://dx.doi org/10.1016/j.epsl.2010.04.004

Söderlund U., Patchett J.P., Vervoort J.D., Isachsen C.E. 2004. The 176Lu decay constantdetermined by $\mathrm{Lu}-\mathrm{Hf}$ and $\mathrm{U}-\mathrm{Pb}$ isotope systematics of Precambrian mafic intrusions. Earth and Planetary Science Letters, 219(34):311-324. https://doi.org/10.1016/S0012-821X(04)00012-3

Stacey J.S. \& Kramers J.D. 1975. Approximation of terrestrial lead isotope evolution by a two-stage model. Earth Planet Science Letters, 26:207-221.

Sun S. \& McDonough W.F. 1989. Chemical and isotopic systematics of oceanic basalts: implications for mantle composition and processes. In Saunders A.D., Norry M.J. (eds.), Magmatism in the Ocean Basins. London, Geological Society, p. 313-345.

Tanaka T., Togashi S., Kamioka H., Amakawa H., Kagami H., Hamamoto T.,Yuhara M., Orihashi Y., Yoneda S., Shimizu H., Kunimaru T., Takahashi K., Yanagi T., Nakano T., Fujimaki H., Shinjo R., Asahara Y., Tanimizu M., Dragusanu C. 2000. JNdi-1: a neodymium isotopic reference in consistency with Lajolla neodymium. Chemical Geology, 168:279-281. http://dx.doi. org/10.1016/S0009-2541(00)00198-4

Tedeschi M., Pedrosa-Soares A.C., Dussin I., Lanarid P., Novo T., Pinheiro M.A., Lana C., Peters D. 2018. Protracted zircon geochronological record of UHT garnet-free granulites in the Southern Brasília orogen (SE Brazil): Petrochronological constraints on magmatism and metamorphism. Precambrian Research, 316:101-126. http://dx.doi.org/10.1016/j.precamres.2018.07.023

Trouw R.A.J., Heilbron M., Ribeiro A., Valeriano C., Paciullo F., Almeida J.C.H., Tupinambá M. 2000. The Central Segment of the Ribeira belt. In: Cordani U., Milani E., Thomaz-Filho A., Campos D. (eds.). Geotectonics of South America. Rio de Janeiro, CPRM, v. 1, p. 287-310.

Trouw R.A.J., Peternel R., Ribeiro A., Heilbron M., Vinagre R., Duffles P., Trouw C.C., Fontainha M., Kussama H.H. 2013. A new interpretation for the interference zone between the southern Brasilia belt and the central Ribeira belt, SE Brazil. Journal of South American Earth Sciences, 48:43-57.

Valeriano C.M., Machado N., Simonetti A., Valladares C.S., Seer H.J., Simões L.S.A. 2004. U Pb geochronology of the southern Brasília belt (SEBrazil): sedimentary provenance, Neoproterozoic orogeny and assembly of West Gondwana. Precambrian Research, 130(1/4):27-55.

Valeriano C.M., Vaz G.S., Medeiros S.R., Neto C.C.A., Ragatky C.D. 2008 The Neodymium isotope composition of the JNdi-1 oxide reference material: results from the LAGIR Laboratory, Rio de Janeiro. In: South American Symposium on Isotope Geology, 6., San Carlos de Bariloche, Argentina. Proceedings ... p. 1-2. 1 CD-ROM.

Valladares C.S., Machado N., Heilbron M., Duarte B.P., Gauthier G. 2008. Sedimentary provenance in the central Ribeira belt based on laser-ablation ICPMS ${ }^{207} \mathrm{~Pb} /{ }^{206} \mathrm{~Pb}$ zircon ages. Gondwana Research, 13:516-526. http:// dx.doi.org/10.1016/j.gr.2007.05.013

Valladares C.S., Machado N., Heilbron M., Gauthier G. 2004. Ages of detrital zircon from siliciclastic successions south of the São Francisco Craton, Brazil implications for the evolution of Proterozoic basin. Gondwana Research, 7(4):913-921. https://doi.org/10.1016/S1342-937X(05)71074-1

Xia L. \& Li X. 2019. Basalt geochemistry as a diagnostic indicator of tectonic setting. Gondwana Research, 65:43-67. http://dx.doi.org/10.1016/j. gr.2018.08.006

Westin A. \& Campos Neto M.C. 2013. Provenance and tectonic setting of the external nappe of the Southern Brasília Orogen. Journal of South American Earth Sciences, 48:220-239. http://dx.doi.org/10.1016/j. jsames.2013.08.006

Wilson M. 1989. Igneous petrogenesis: a global tectonic approach. London, Unwin Hyman, 466 p.

Wise S.A. \& Waters R.L. 2007. Certificate of analysis standard reference material ${ }^{\circledR} 987$ Strontium Carbonate (Isotopic Standard). NIST National Institute of Standards \& Technology, 2 p. Available at: $<$ https://www.nist.gov>

Zeh A., Gerdes A., Will T.M., Frimmel H.E. 2010. Hafnium isotope homogenization during metamorphic zircon growth in amphibolite-facies rocks: examples from the Shackleton Range (Antarctica). Geochimica et Cosmochimica Acta, 74(16):4740-4758. http://dx.doi.org/10.1016/j. gca.2010.05.016 